\title{
Observable effects of general new scalar particles
}

\author{
J. de Blas, ${ }^{a}$ M. Chala, ${ }^{b}$ M. Pérez-Victoria ${ }^{c}$ and J. Santiago ${ }^{c, d}$ \\ ${ }^{a}$ INFN, Sezione di Roma, \\ Piazzale A. Moro 2, Rome, I-00185 Italy \\ ${ }^{b} D E S Y$, \\ Notkestrasse 85, Hamburg, 22607 Germany \\ ${ }^{c}$ Departamento de Física Teórica y del Cosmos and CAFPE, Universidad de Granada, \\ Campus de Fuentenueva, Granada, E-18071 Spain \\ ${ }^{d}$ Theory Division, CERN, \\ Geneva 23, CH1211 Switzerland \\ E-mail: Jorge.DeBlasMateo@roma1.infn.it, mikael.chala@desy.de, \\ mpv@ugr.es, jsantiago@ugr.es
}

ABSTRACT: We classify all possible new scalar particles that can have renormalizable linear couplings to Standard Model fields and therefore be singly produced at colliders. We show that this classification exhausts the list of heavy scalar particles that contribute at the tree level to the Standard Model effective Lagrangian to dimension six. We compute this effective Lagrangian for a general scenario with an arbitrary number of new scalar particles and obtain flavor-preserving constraints on their couplings and masses. This completes the tree-level matching of the coefficients of dimension five and six operators in the effective Lagrangian to arbitrary extensions of the Standard Model.

Keywords: Beyond Standard Model, Phenomenological Models

ARXIV EPRINT: 1412.8480 


\section{Contents}

1 Introduction 1

2 Standard model extensions with extra scalar fields 3

3 The effective Lagrangian for heavy new scalar particles 5

4 Observable effects of new scalars $\quad 8$

5 Precision constraints on new scalars 11

6 Scalar extensions with other new particles 15

$\begin{array}{llr}7 & \text { Conclusions } & 18\end{array}$

$\begin{array}{ll}\text { A Basis of dimension-six operators } & 20\end{array}$

B Operator coefficients in the effective Lagrangian 23

\section{Introduction}

The discovery of the Higgs boson at the Large Hadron Collider (LHC) [1, 2] has opened a new era in particle physics in which we have, for the first time, direct access to the electroweak symmetry breaking sector. The naturalness problem associated to this scalar sector is still one of the main reasons to expect new physics beyond the Standard Model (SM) at the TeV scale, and thus accessible to the LHC. However, the lack of significant deviations from the SM predictions after Run 1 suggests that, even if really present, the new particles may be too heavy to be produced on-shell, so that only their indirect effects can be observed at the LHC - although it is certainly possible that they are just above the current reach and can still be directly produced at the higher energies of Run 2 . In such a case, the natural language to parameterize the expected effects of new physics is that of effective theories.

Effective Lagrangians provide a model-independent description of the effects of new particles at energies much smaller than their masses. Hence, they are the perfect tool to study any new physics that lies beyond the reach of our current experiments. A single higher-dimensional gauge-invariant operator contributes in general to several different couplings between SM particles after electroweak symmetry breaking, leading to non-trivial correlations between different observables [3]. These correlations can be tested experimentally or, alternatively, be used to predict the size of the expected deviations with respect to the SM predictions [4]. In addition, higher-dimensional operators are often generated 
by a smaller number of couplings in specific ultraviolet completions and therefore the coefficients of different operators are correlated as well. It could be argued that these latter correlations are model-dependent, defeating the very purpose of the effective Lagrangian. However, model independence can be recovered if a complete dictionary between ultraviolet completions and effective operators is built. Such a dictionary would provide a comprehensive classification of new physics with potentially observable effects at the LHC. This classification can hence guide experimental searches to ensure that no viable option is missed at the LHC, and help to identify the origin of possible deviations from the SM predictions.

Encouraged by the recent experimental observation of a Higgs sector, in this article we focus on new scalar particles. We first classify all the possible new scalars that can couple linearly, with renormalizable interactions, to SM fields, and write their most general phenomenologically-relevant interactions. These particles can be singly produced at colliders with sizable couplings and have therefore the most promising discovery potential at the LHC. The corresponding general interactions of new quarks, leptons and vector bosons have already been given in refs. [5, 6] and [7], respectively. Our results here thus complete the description of arbitrary new particles with linear gauge-invariant renormalizable couplings to the SM fields. This provides an extremely useful scheme for a model-independent interpretation of LHC searches. Particular models correspond to specific choices of the general couplings and masses in this set-up. Therefore, once the experimental results are written in terms of the general parameters it is straightforward to derive consequences for any model of choice.

It turns out that the same classification also covers all possible new scalar particles that contribute, once integrated out at the tree level, to the SM effective Lagrangian of dimension five and six. We perform this integration explicitly. In predictive models loop contributions are suppressed, so it is expected that the leading observable consequences of new heavy scalars are those generated at tree level. Except for this assumption, our results are completely general and can be used for an arbitrary extension of the SM with heavy scalars, independently of their amount and quantum numbers. Furthermore, extra heavy particles with different spins do not mix with the heavy scalars in their contribution to the dimension-six effective Lagrangian at tree level. Therefore, together with the effective Lagrangians generated by the most general extension of the SM with new quarks [5], leptons [6] and vector bosons [7], our results complete the tree-level dictionary between any model of new physics and the dimension-six SM effective Lagrangian. This dictionary can be used to trivially obtain the observable implications of an arbitrary model of new physics at energies much smaller than the masses of the new particles involved. It is also a powerful tool to investigate correlations or cancellations predicted among observables in specific extensions of the SM. Finally, it provides a rationale for the calculation of the constraints on the coefficients of the SM effective Lagrangian, as one can put constraints on the sources of the effective operators in a correlated way rather than on arbitrary combinations of them.

The article is organized as follows. We classify in section 2 all possible new scalars that can have linear interactions with SM fields. We describe in section 3 how to compute 
the effective Lagrangian that results from the tree-level integration of an arbitrary number and type of new heavy scalars, and show that the previous classification exhausts the list of new spin-0 particles that can contribute to the dimension-six effective Lagrangian at tree level. In section 4 we discuss what effects induced by the heavy scalars are observable at this order. Some applications of the effective Lagrangian are presented in section 5 . Finally, we comment on the interplay between new scalars and particles with different spin in section 6 , and conclude in section 7 . The basis of dimension-six operators and all the relevant scalar interactions with the resulting effective Lagrangians, are given in appendix A and B, respectively.

\section{Standard model extensions with extra scalar fields}

We consider a general renormalizable theory for extra scalars and SM fields, invariant under Lorentz transformations and under the complete $\mathrm{SU}(3)_{c} \otimes \mathrm{SU}(2)_{L} \otimes \mathrm{U}(1)_{Y}$ gauge group. The new scalar fields will come in complete representations of this group, which can be decomposed into their irreducible components $\sigma$. Non-renormalizable interactions are also possible in principle, but in a predictive theory they will be suppressed by a scale larger than the mass of the extra scalars. Here we concentrate on the leading effects, which are generically described by operators of dimension four at most.

The most general Lagrangian for such an extension of the SM can be written as

$$
\mathcal{L}=\mathcal{L}_{\mathrm{SM}}+\mathcal{L}_{\sigma}+\mathcal{L}_{\text {int }},
$$

where $\mathcal{L}_{\mathrm{SM}}$ is the SM Lagrangian, $\mathcal{L}_{\sigma}$ contains the kinetic (with covariant derivatives) and mass terms for the new scalars and $\mathcal{L}_{\text {int }}$ describes the non-gauge interactions of the extra scalars.

The SM Lagrangian reads, in standard notation ${ }^{1}$

$$
\begin{aligned}
\mathcal{L}_{\mathrm{SM}}= & -\frac{1}{4} G_{\mu \nu}^{A} G^{A \mu \nu}-\frac{1}{4} W_{\mu \nu}^{a} W^{a} \mu \nu-\frac{1}{4} B_{\mu \nu} B^{\mu \nu} \\
& +\overline{l_{L}^{i}} i \not D l_{L}^{i}+\overline{q_{L}^{i}} i \not D q_{L}^{i}+\overline{e_{R}^{i}} i \not D e_{R}^{i}+\overline{u_{R}^{i}} i \not D u_{R}^{i}+\overline{d_{R}^{i}} i \not D d_{R}^{i} \\
& +\left(D_{\mu} \phi\right)^{\dagger} D^{\mu} \phi-U(\phi)-\left(y_{i i}^{e} \overline{l_{L}^{i}} \phi e_{R}^{i}+y_{i i}^{d} \overline{q_{L}^{i}} \phi d_{R}^{i}+V_{i j}^{\dagger} y_{j j}^{u} \overline{q_{L}^{i}} \tilde{\phi} u_{R}^{j}+\text { h.c. }\right) .
\end{aligned}
$$

In order to fix the meaning of flavor indices, we have chosen a basis in which the Yukawa interactions for the charged-leptons and down-type quarks are diagonal. As usual, $\tilde{\phi}=$ $i \sigma_{2} \phi^{*}$ denotes the iso-doublet of hypercharge $-1 / 2$, constructed with the Higgs doublet $\phi$. The Higgs scalar potential is

$$
U(\phi)=-\mu_{\phi}^{2}|\phi|^{2}+\lambda_{\phi}|\phi|^{4} .
$$

The Lagrangian $\mathcal{L}_{\sigma}$ contains the gauge-invariant kinetic and mass terms for the new scalars:

$$
\mathcal{L}_{\sigma}=\sum_{\sigma} \eta_{\sigma}\left[\left(D_{\mu} \sigma\right)^{\dagger} D^{\mu} \sigma-M_{\sigma}^{2} \sigma^{\dagger} \sigma\right]
$$

\footnotetext{
${ }^{1}$ We use capital indices $A, B, C$ as color indices, whereas lower case indices $a, b=1,2,3$ tag fields in the adjoint of $\mathrm{SU}(2)_{L}$. Latin indices $i, j, k$ are used to label different generations.
} 
where $\eta_{\sigma}=1, \frac{1}{2}$, for complex and real scalars, respectively. Note that we are working in a basis with canonical kinetic terms and diagonal mass matrices for all scalar fields, including the Higgs doublet. To match models written in a different basis, the diagonalization must be performed prior to using our formulas. ${ }^{2}$

Finally, $\mathcal{L}_{\text {int }}$ contains the renormalizable interactions of the extra scalars (among themselves and with the SM fields), except for the gauge interactions, which are already included in $\mathcal{L}_{\sigma}$. We can distinguish between interactions with fermions and purely scalar interactions:

$$
\mathcal{L}_{\text {int }}=-V(\{\sigma\}, \phi)-\sum_{\sigma} \eta_{\sigma}\left(\sigma^{\dagger} J_{\sigma}+\text { h.c. }\right)
$$

The chirality-flipping fermionic currents $J_{\sigma} \sim \overline{\psi_{L}} \otimes \xi_{R}$ or $J_{\sigma} \sim \overline{\xi_{R}} \otimes \psi_{L}$ couple to one scalar field with a dimensionless coupling. The potential $V$ contains scalar interactions between the new particles and, possibly, the SM Higgs fields. Together with the mass terms and $U$, it forms the total scalar potential. As explained above, $V$ does not include mass mixing terms, since we work in a basis with diagonal quadratic terms. Thus, each term in $V$ contains either three or four scalars, with couplings of dimension one or zero, respectively. Furthermore, all the terms in $V$ have at least one $\sigma$ field.

The new scalars with linear interactions in $\mathcal{L}_{\text {int }}$ can be singly produced at tree level in colliders. ${ }^{3}$ By "linear interactions" we mean that $\mathcal{L}_{\text {int }}$ contains some non-vanishing term that is the product of SM fields and a single power of the given extra scalar field, with no other extra scalars. Gauge invariance and the particle content of the SM strongly constraints the quantum numbers of new scalars that can have such linear interactions. As we show in the next section, only the scalars in representations that allow for these linear couplings can contribute at tree level, to order $1 / M_{\sigma}^{2}$ in the heavy mass limit, to observable processes with SM particles in the initial and final states. We list in table 1 all the irreducible representations of scalars with linear interactions of this kind. The scalar representations $\mathcal{S}, \Xi_{0}, \Theta_{1}$ and $\Theta_{3}$ do not couple to the SM fermions. In appendix B we write the interactions in $\mathcal{L}_{\text {int }}$ explicitly, including only those that have an impact in the SM effective Lagrangian at dimension six. These include the mentioned linear interactions of the scalar fields in the irreducible representations of table 1 , as well as terms involving two or three scalar fields in the same set of representations. Note that, in particular, new scalars can always couple with the SM through a Higgs-portal type of coupling $\left(\sigma^{\dagger} \sigma\right)\left(\phi^{\dagger} \phi\right)$ [8]. However, only the ones in our list with the right quantum numbers to allow for linear couplings to the SM will induce dimension-six operators when integrated at tree level. Moreover, as we explain in the next section, interactions involving two or more new scalars are relevant for the calculation of the effective Lagrangian to dimension six only in those

\footnotetext{
${ }^{2}$ Furthermore, we assume that there are no tadpole operators in the electroweak symmetric phase. This entails no loss of generality since these tadpoles, which are only possible for new singlet scalars, can always be eliminated by a shift of the singlet field(s). The only effect of this shift is a redefinition of the parameters that we write explicitly.

${ }^{3}$ Other scalar fields can also be singly produced if they mix with these after electroweak symmetry breaking, but if they are heavy the production rate will be suppressed by the square of small mixings.
} 


\begin{tabular}{|lcccccccc|}
\hline $\begin{array}{l}\text { Colorless } \\
\text { Scalars }\end{array}$ & $\mathcal{S}$ & $\mathcal{S}_{1}$ & $\mathcal{S}_{2}$ & $\varphi$ & $\Xi_{0}$ & $\Xi_{1}$ & $\Theta_{1}$ & $\Theta_{3}$ \\
\hline Irrep & $(1,1)_{0}$ & $(1,1)_{1}$ & $(1,1)_{2}$ & $(1,2)_{\frac{1}{2}}$ & $(1,3)_{0}$ & $(1,3)_{1}$ & $(1,4)_{\frac{1}{2}}$ & $(1,4)_{\frac{3}{2}}$ \\
\hline \hline $\begin{array}{l}\text { Colored } \\
\text { Scalars }\end{array}$ & $\omega_{1}$ & $\omega_{2}$ & $\omega_{4}$ & $\Pi_{1}$ & $\Pi_{7}$ & $\zeta$ & & \\
\hline Irrep & $(3,1)_{-\frac{1}{3}}$ & $(3,1)_{\frac{2}{3}}$ & $(3,1)_{-\frac{4}{3}}$ & $(3,2)_{\frac{1}{6}}$ & $(3,2)_{\frac{7}{6}}$ & $(3,3)_{-\frac{1}{3}}$ & & \\
\hline \hline $\begin{array}{l}\text { Colored } \\
\text { Scalars }\end{array}$ & $\Omega_{1}$ & $\Omega_{2}$ & $\Omega_{4}$ & $\Upsilon$ & $\Phi$ & & & \\
\hline Irrep & $(6,1)_{\frac{1}{3}}$ & $(6,1)_{-\frac{2}{3}}$ & $(6,1)_{\frac{4}{3}}$ & $(6,3)_{\frac{1}{3}}$ & $(8,2)_{\frac{1}{2}}$ & & & \\
\hline
\end{tabular}

Table 1. Scalar bosons with linear renormalizable interactions with the SM fields. The quantum numbers $\left(R_{c}, R_{L}\right)_{Y}$ denote the irreducible representation (Irrep) $R_{c}$ under $\mathrm{SU}(3)_{c}, R_{L}$ under $\mathrm{SU}(2)_{L}$ and the hypercharge $Y$, respectively. The hypercharge is normalized such that the electric charge is $Q=Y+T_{3}$. Looking only at the quantum numbers, some readers might miss in this list a scalar particle transforming as a $(1,2)_{\frac{3}{2}}$ and coupling linearly to three Higgs doublets. However, the corresponding operator actually vanishes, since it involves an antisymmetric combination of the Higgs fields.

cases where the scalar potential contains trilinear couplings of new scalars to two Higgs fields (see tables 9, 13, 14 and 28 in appendix B).

Extensions of the SM with new scalar fields have been extensively considered in the past and partial classifications have been presented, with special emphasis on their effect on baryon and lepton number violation [9-11], collider physics [12,13], top physics [14] or flavor physics [15]. However the complete classification of the scalar fields that can couple linearly to the SM and the calculation of the tree-level dimension-six effective Lagrangian for the most general extension of the SM with an arbitrary number of new scalar fields has, to the best of our knowledge, never been presented before.

\section{The effective Lagrangian for heavy new scalar particles}

In this paper we are mainly interested in the effects of heavy new scalars, with masses $M_{\sigma}$ that are large in comparison to the Higgs vacuum expectation value (vev) and to the energies probed by the available experimental data. An efficient way to describe these effects at leading order is to integrate the heavy scalars out and expand in $1 / M_{\sigma}$ to obtain an effective Lagrangian with gauge-invariant local operators of dimension up to six. This allows for a direct comparison with model-independent analyses and also for a simple combination with other extensions of the SM. In our theory with general extra scalars, the coefficients of these operators will be simple functions of the couplings and masses of the heavy scalars. Once again, we work at the tree level for simplicity and to avoid further suppressions (see [13, 16-18] for examples of one-loop integration of scalar multiplets).

In this section we describe the integration of the heavy scalar fields. In particular, we show that among the infinite scalar representations that can appear in $\mathcal{L}_{\text {int }}$, only the 
ones in table 1 contribute to the effective Lagrangian to dimension six. For clarity, our discussion will be slightly schematic. The complete explicit results, i.e. the dimension-six operators and the values of their coefficients, are collected in the appendices.

In the following we assume that, before electroweak symmetry breaking, the scalar mass matrix has only one negative eigenvalue, $-\mu_{\phi}^{2}$. The eigenvector is a $(1,2)_{1 / 2}$ scalar field $\phi$, which we identify with the SM scalar doublet. The other eigenvalues, $M_{\sigma}^{2}$, are assumed to be large in comparison with the Higgs vev and with the relevant energies. Finally, we assume that the dimensionful couplings $\kappa_{\sigma}$ that multiply the dimension-three operators in $V$ are at most of the size of the smaller heavy-scalar mass, $\left|\kappa_{\sigma}\right| \lesssim M$, with $M=\operatorname{Min}\left\{\left|M_{\sigma}\right|\right\}$. These assumptions are well motivated by the agreement, within the available precision, of experimental results and SM predictions for Higgs observables. They lead to a decoupling scenario and allow us to perform the integration in the electroweak symmetric phase, which is extremely convenient. The occurrence of electroweak symmetry breakdown and all its effects are captured to order $1 / M^{2}$ by the effective Lagrangian. This includes the case in which the extra scalars acquire (suppressed) vevs in the Higgs phase [16, 17].

The integration at the tree level can be performed solving the classical equations of motion for the heavy fields and inserting the solutions into the original Lagrangian. This procedure manifestly preserves the gauge invariance of the original theory. Let $\sigma^{i}$ be each of the scalar fields. Different values of the index $i$ label different scalars, in the same or in different representations. We will use upper and lower indices for the fields and their complex conjugates, respectively. The covariant propagator is

$$
\Delta_{i}=-\left(D_{i}^{2}+M_{i}^{2}\right)^{-1}=-\frac{1}{M_{i}^{2}}\left(1-\frac{D_{i}^{2}}{M_{i}^{2}}\right)+O\left(1 / M^{6}\right),
$$

with $D_{i}$ the covariant derivative acting on $\sigma^{i}$. The part of the Lagrangian that contains the extra scalars reads

$$
\eta_{(i)} \sigma_{i}^{\dagger} \Delta_{(i)}^{-1} \sigma^{i}+\mathcal{L}_{\text {int }}
$$

We are using the convention of repeated indices, contracting upper and lower indices. A parenthesis indicates an index that can run but does not count as repeated to induce the running (indices in parenthesis actually refer to the diagonal elements of a diagonal matrix, the propagator). The interaction Lagrangian is a polynomial in $\sigma$ of degree 4 with no constant term. Hence, it will be of the form

$$
\mathcal{L}_{\text {int }}=-\sum_{m+n=1}^{4} \sigma_{j_{1}}^{\dagger} \cdots \sigma_{j_{n}}^{\dagger} W_{i_{1} \ldots i_{m}}^{j_{1} \ldots j_{n}} \sigma^{i_{1}} \cdots \sigma^{i_{m}},
$$

where $m$ and $n$ vary independently subject to the indicated constraint. The operators $W_{i_{1} \ldots i_{m}}^{j_{1} \ldots j_{n}}$ are formed with SM fields only and obey the obvious hermiticity conditions inherited from $\mathcal{L}_{\text {int }}$. In general, they carry reducible representations of the gauge group, but the operators with one index $i$ belong to the irreducible representation of the associated $\sigma^{i}$ or $\sigma_{i}^{\dagger}$. The equations of motion for the new scalars read

$$
\sigma^{i}=-\Delta_{(i)} \frac{\partial \mathcal{L}_{\text {int }}}{\partial \sigma_{i}^{\dagger}}
$$



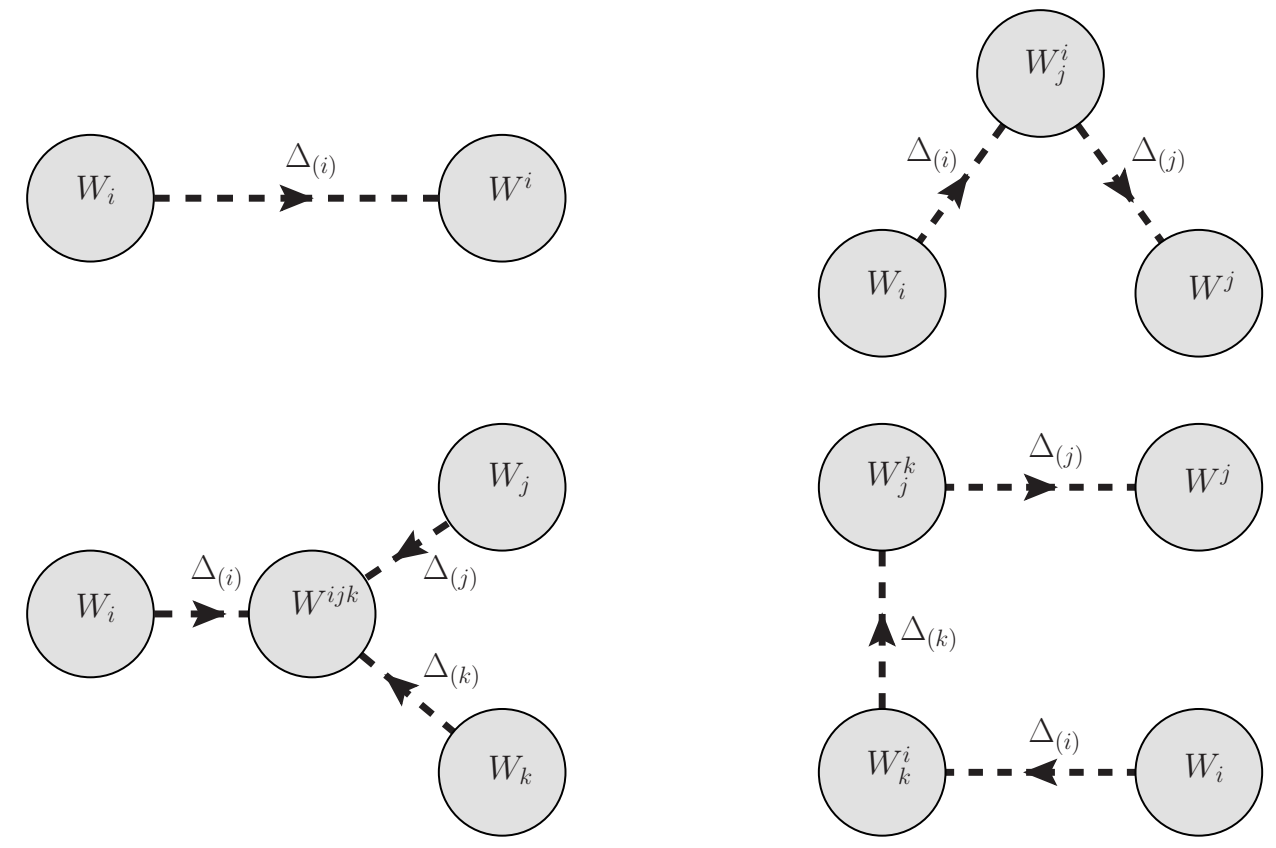

Figure 1. Feynman diagrams contributing to the effective Lagrangian to order $1 / M^{2}$. Nonequivalent permutations of the arrow directions shown here should be considered as well.

Note that the right-hand sides depend on the fields $\sigma$, so these are not explicit solutions. But the equations can be solved iteratively. The crucial point is that $\Delta_{i}$ is $O\left(1 / M^{2}\right)$, whereas the couplings in $\mathcal{L}_{\text {int }}$ are either dimensionless or $O(M)$, at most. Therefore, the iterative solution of (3.4) starts at $O(1 / M)$, and each correction (step of the iteration) is suppressed by at least another $1 / M$ factor. Using (3.3) in the right-hand side of (3.4), we can perform the iteration explicitly. Then we plug the iterative solution in (3.2) and (3.3), neglecting $O\left(1 / M^{3}\right)$ terms, to obtain

$$
\begin{aligned}
\mathcal{L}_{\text {eff }}= & \mathcal{L}_{\mathrm{SM}}-\Delta_{(i)} W_{i} W^{i}-\Delta_{(i)} \Delta_{(j)}\left(W_{i j} W^{i} W^{j}+W^{i j} W_{i} W_{j}+W_{j}^{i} W_{i} W^{j}\right) \\
& -\Delta_{(i)} \Delta_{(j)} \Delta_{(k)}\left(W^{i j k} W_{i} W_{j} W_{k}+W_{i j k} W^{i} W^{j} W^{k}+W_{k}^{i j} W_{i} W_{j} W^{k}+W_{j k}^{i} W_{i} W^{j} W^{k}\right. \\
& \left.+2 W^{i k} W_{k}^{j} W_{i} W_{j}+2 W_{i k} W_{j}^{k} W^{i} W^{j}+4 W^{i k} W_{k j} W_{i} W^{j}+W_{k}^{i} W_{j}^{k} W_{i} W^{j}\right) \\
& +O\left(1 / M^{3}\right) .
\end{aligned}
$$

In each term, $\Delta_{(i)}$ acts on the operator with an upper index $i$. The covariant propagators $\Delta_{i}$ are to be expanded in $1 / M_{i}^{2}$, as in (3.1). This result matches precisely the one obtained from the possible Feynman diagrams with heavy-scalar propagators that contribute to order $1 / M^{2}$, shown in figure 1 . The blobs in this figure represent the SM operators $W_{j_{1} \ldots j_{n}}^{i_{1} \ldots i_{m}}$ with $m$ incoming and $n$ outgoing lines, and the arrowed lines represent the covariant propagators $\Delta_{i}$.

Observe that in all the terms in (3.5) except the ones in the last line, there is one $W_{i}$ or $W^{i}$ operator for each propagator index $i . W_{i}$ and $W^{i}$ arise from terms in $\mathcal{L}_{\text {int }}$ with only one heavy scalar $\left(\sigma^{i}\right.$ or $\left.\sigma_{i}^{\dagger}\right)$, which are what we have called linear interactions. In the 
last line (corresponding to the last Feynman diagram), on the other hand, all terms have a propagator with index $k$ that is not attached to any $W_{k}$ or $W^{k}$, but only to operators with two indices. However, these terms are actually the contraction of the two one-index operators $\widetilde{W}_{k}=\left[W_{k j} \Delta_{(j)} W^{j}\right]$ and $\widetilde{W}^{k}=\left[W^{k j} \Delta_{(j)} W^{j}\right]$ (or variations in the position of the indices). The operators $\widetilde{W}_{k}$ and $\widetilde{W}^{k}$ are in the same gauge representation as $W_{k}$ and $W^{k}$, respectively. Moreover, to allow for an $O\left(1 / M^{2}\right)$ contribution, the operators $\widetilde{W}_{k}$ and $\widetilde{W}^{k}$ must have a dimensionless coefficient and, hence, scaling dimension four. Therefore, the scalars $\sigma^{k}\left(\sigma_{k}^{\dagger}\right)$ associated to $\widetilde{W}_{k}\left(\widetilde{W}^{k}\right)$ must also belong to a representation that allows for renormalizable linear interactions. We conclude that only the scalar fields in the irreducible representations of table 1 contribute at the tree level to the effective Lagrangian to dimension six.

Note also that the last two topologies in figure 1 only contribute to this order when the four blobs contain $O(M)$ dimensionful couplings, which requires that all of them arise from trilinear terms in the scalar potential. In particular, the SM fermions only appear through the diagrams with the first and second topologies. Gauge bosons only arise at dimension six from the covariant propagator of the first topology, when both blobs represent trilinear interactions in the potential.

A complete basis of gauge-invariant operators to dimension six, including the ones generated by heavy scalars, is given in appendix A. The relevant interactions of arbitrary scalars and the detailed results of the integration are collected in appendix B. ${ }^{4}$

\section{Observable effects of new scalars}

The leading indirect effects of new heavy scalar particles on physical observables are described by the dimension five and six effective operators, with coefficients given in tables 928 in appendix B. In this section we give an overview of these effects. We discuss colored and colorless scalars in turn.

The scalar fields with $\mathrm{SU}(3)_{c}$ quantum numbers manifest themselves, at dimension six, only through four-fermion interactions. Therefore they can be tested in two-to-two fermion processes or in particle decays. The flavor structure of the scalar interactions makes them particularly sensitive to constraints from flavor-violating processes, although it is always possible to go into an alignment limit in which each new scalar couples exclusively to certain fermion generations, up to factors of the Cabibbo-Kobayashi-Maskawa (CKM) matrix. In such a case, the bounds from flavor-preserving processes can become dominant. We will only consider this scenario in the following.

The scalar fields $\omega_{1}, \omega_{4}, \Pi_{1}, \Pi_{7}$ and $\zeta$ carry quantum numbers that allow for leptonquark Yukawa interactions. These scalar leptoquarks can be tested in the dilepton processes $e^{+} e^{-} \rightarrow$ had at LEP2 and $p p \rightarrow \ell^{+} \ell^{-}$at the LHC, where the new particles are exchanged in the $t$ channel, and also in low-energy experiments (e.g. parity violation in atoms). The multiplets $\omega_{1}, \omega_{4}$ and $\zeta$ admit purely hadronic interactions too. The simultaneous presence of all these interactions introduces a violation of lepton $(L)$ and baryon $(B)$ number, as is

\footnotetext{
${ }^{4}$ In some cases, we employ algebraic identities and/or field redefinitions to transform the induced operators into the ones in our basis.
} 
manifest by the generation of the operators labeled as " $B$ \& $L$ " in tables 19, 17, and 22. These contributions are proportional to the product of one lepton-quark and one quarkquark Yukawa coupling, so the strong constraints set by the non-observation of proton decay forces one of these two couplings to be very small (see for instance [19]).

The remaining colored representations, $\omega_{2}, \Omega_{1}, \Omega_{2}, \Omega_{4}, \Upsilon$ and $\Phi$, only admit quark Yukawa interactions and therefore generate only four-quark contact interactions. Aside from flavor observables, these can be tested in dijet production at hadron colliders. If coupled to the first and third family, top pair production (with opposite or same sign) is also possible.

The phenomenology of colorless scalars is significantly richer. Again, the exchange of some colorless heavy scalars generates four-fermion operators, which in this case include operators with four leptons. In particular, all the four-fermion interactions induced by the multiplets $\mathcal{S}_{1,2}$ and $\Xi_{1}$ are purely leptonic. The effects of these operators could show up in the $e^{+} e^{-} \rightarrow \ell^{+} \ell^{-}$data taken at LEP2 or in low-energy experiments such as measurements of parity violation in Møller scattering. ${ }^{5}$ In general, these four-lepton operators also contribute to very sensitive lepton flavor violating processes, such as $\mu^{-} \rightarrow e^{+} e^{-} e^{-}$(note that this cannot be mediated by $\mathcal{S}_{1}$ ) or $\tau^{-} \rightarrow \mu^{-} e^{+} e^{-}$. But similarly to the quark case, these dangerous effects are absent when the scalar couplings are properly aligned with the SM lepton flavors.

Colorless scalars can also have visible effects in other types of observables. To start with, the hypercharge-one iso-triplet $\Xi_{1}$ is the only scalar multiplet that can produce leptonnumber violation. Indeed, it contributes to the dimension five Weinberg operator [21] $\mathcal{O}_{5}=\overline{l_{L}^{c}} \tilde{\phi}^{*} \tilde{\phi}^{\dagger} l_{L}$, which generates Majorana masses for the SM neutrinos. This is nothing but the well-known seesaw mechanism of type II. Unless the scalar iso-triplet is very heavy (and thus does not contribute to other observables), the smallness of neutrino masses requires that either the lepton Yukawa couplings of the scalar or its linear interactions with the Higgs be tiny [22-24].

Colorless scalars contribute to the following three purely bosonic dimension-six operators: $\mathcal{O}_{\phi \square}=\left(\phi^{\dagger} \phi\right) \square\left(\phi^{\dagger} \phi\right), \mathcal{O}_{\phi D}=\left(\phi^{\dagger} D_{\mu} \phi\right)\left(D^{\mu} \phi^{\dagger} \phi\right)$ and $\mathcal{O}_{\phi}=\left(\phi^{\dagger} \phi\right)^{3} / 3$. The first one, $\mathcal{O}_{\phi D}$, is in one to one correspondence with the Peskin-Takeuchi oblique $T$ parameter [25],

$$
T=-\frac{\alpha_{\phi D}}{2 \alpha_{e m}} \frac{v^{2}}{\Lambda^{2}}
$$

which is very strongly constrained by electroweak precision data (EWPD), especially now that the value of the Higgs mass is known. Only the colorless iso-triplets $\Xi_{0}$ and $\Xi_{1}$ contribute to $O_{\phi D}$ at the tree level. Hence, these are the only scalars whose effects break custodial symmetry at tree level, to dimension six in the effective Lagrangian expansion. In this regard, note that, unlike the weak iso-singlets and iso-doublets, quadruplets do also break custodial isospin at the tree level if they acquire a vev. However, because of the absence of trilinear interactions with two Higgs fields, this vev is suppressed by a factor

\footnotetext{
${ }^{5}$ Four-lepton operators not involving electrons could in principle be accessible through four-lepton production at the LHC or ILC. The sensitivity is however only marginal if there is no resonant production [20].
} 
of $O\left(1 / M^{2}\right)$. Therefore, quadruplet custodial isospin breaking effects appear starting at dimension eight.

Both $\mathcal{O}_{\phi D}$ and $\mathcal{O}_{\phi \square}$ renormalize the wavefunction of the physical Higgs field,

$$
H \rightarrow\left(1+\alpha_{\phi \square} \frac{v^{2}}{\Lambda^{2}}-\frac{1}{4} \alpha_{\phi D} \frac{v^{2}}{\Lambda^{2}}\right) H,
$$

and therefore enter in most Higgs observables. Nevertheless, the constraints on the coefficient of $\mathcal{O}_{\phi D}$ from EWPD are significantly stronger than the ones from Higgs physics [26, 27 , and completely dominate in the global fit. The operator $\mathcal{O}_{\phi}$ introduces corrections to the Higgs vev and mass parameters, which can always be absorbed in the physical values. It also gives a direct contribution to the self-coupling of the physical Higgs,

$$
\Delta \mathcal{L}_{H^{3}}=\frac{5}{6} \alpha_{\phi} \frac{v^{3}}{\Lambda^{2}} H^{3},
$$

which is in principle observable in Higgs pair production. However, the LHC data at $8 \mathrm{TeV}$ are not sensitive enough to probe the Higgs self-coupling. Given the small cross sections for Higgs pair production in the SM, a measurement of the Higgs self-coupling seems to be challenging even with the results of Run 2 , and may require of the high-luminosity upgrade of the LHC [28]. On the other hand, a relatively large enhancement in diHiggs production due to the effect of $\mathcal{O}_{\phi}$ could unveil the presence of new physics effects before sensitivity to the SM coupling is attained.

With the exception of $\mathcal{S}_{1}$ and $\mathcal{S}_{2}$, all the colorless multiplets contribute to Higgs observables (the singlet $\mathcal{S}$ and the two quadruplets $\Theta_{1}$ and $\Theta_{2}$ only contribute to Higgs observables). The singlet $\mathcal{S}$ and the iso-triplets $\Xi_{0}$ and $\Xi_{1}$ contribute to $\mathcal{O}_{\phi \square}$ and their trilinear couplings can therefore be constrained by Higgs measurements. The latter two, however, contribute to $\mathcal{O}_{\phi D}$ via the same trilinear coefficients, which are therefore constrained mainly by EWPD. The quadruplets $\Theta_{1}$ and $\Theta_{2}$ only contribute to $\mathcal{O}_{\phi}$, so, to dimension six, their effects are not observable in current data.

Finally, the scalar-fermion operators $\mathcal{O}_{e \phi}=\left(\phi^{\dagger} \phi\right)\left(\overline{l_{L}} \phi e_{R}\right), \mathcal{O}_{d \phi}=\left(\phi^{\dagger} \phi\right)\left(\overline{q_{L}} \phi d_{R}\right)$, and $\mathcal{O}_{u \phi}=\left(\phi^{\dagger} \phi\right)\left(\overline{q_{L}} \tilde{\phi} u_{R}\right)$ correct the SM Yukawa interactions:

$$
\Delta \mathcal{L}_{\text {Yukawa }}=\frac{1}{\sqrt{2}} H\left(\left(\alpha_{e \phi}\right)_{i j} \overline{e_{L}^{i}} e_{R}^{j}+\left(V \alpha_{u \phi}\right)_{i j} \overline{u_{L}^{i}} u_{R}^{j}+\left(\alpha_{d \phi}\right)_{i j} \overline{d_{L}^{i}} d_{R}^{j}+\text { h.c. }\right) \frac{v^{2}}{\Lambda^{2}} .
$$

In this equation we have already reabsorbed the corrections to the masses in the definition of the SM Yukawa matrices. These operators are generated by the colorless iso-triplets and iso-doublet. For $\Xi_{0}$ and $\Xi_{1}$, the coefficients are proportional to the (squared) trilinear couplings, which as pointed out above contribute to the $T$ parameter and are strongly constrained by EWPD. In the case of the doublet $\varphi$, the coefficients are proportional to the product of the scalar coupling $\lambda_{\varphi}\left(\varphi^{\dagger} \phi\right)\left(\phi^{\dagger} \phi\right)+$ h.c. and the corresponding fermionic coupling. The Higgs observables only constrain this product but not the individual couplings. (The scalar coupling enters quadratically in the coefficient of $\mathcal{O}_{\phi}$ but, as indicated above, there is no significant bound on this coefficient at present.)

In the next section we give some numerical results for bounds that can be obtained on the couplings and masses of the heavy scalar particles from the available measurements. 


\section{Precision constraints on new scalars}

As explained in the previous section, the effects of the scalar couplings in tables 9-27 can potentially be observed in several different physical processes. The good agreement of the SM predictions with most of the current observations implies bounds on the different interactions. In this section, we use the effective Lagrangian results obtained in the previous sections to derive flavor-conserving limits on some of the scalar representations.

For the sake of simplicity, in the fits presented here we consider only one scalar multiplet at a time and always assume that only one of the possible couplings of each scalar is nonvanishing. ${ }^{6}$ In most cases, this assumption gives rise to conservative limits. More general scenarios are certainly interesting and can be studied with the tools provided in this paper. At any rate, it is important to observe that there are strong phenomenological reasons for not considering certain couplings simultaneously, as we explain next.

As stressed above, the new scalar fermionic interactions do not conserve flavor in general. Thus, they are subject to the constraints imposed by observables measured in flavor-violating processes, which are usually much stronger than the ones from flavorconserving observables. For pure hadronic interactions in the form of four-quark operators, for instance, the observables with $\Delta F=2$ transitions (e.g. $\epsilon_{K}$ or $\Delta m_{K}$, measured in $K^{0}-\overline{K^{0}}$ mixing) impose bounds on the new physics scale typically around $10^{2}-10^{4} \mathrm{TeV}$, assuming order-one couplings [30]. Lepton flavor violating processes also impose strong bounds, especially from rare decays such as $\mu^{-} \rightarrow e^{+} e^{-} e^{-}$or $\mu \rightarrow e \gamma$. Flavor-preserving results are meaningful in scenarios in which flavor constraints are subdominant or do not apply. For instance, flavor constraints can be avoided in a natural manner by enforcing an appropriate symmetry on these SM extensions, which requires extending each scalar gauge multiplet to a full multiplet under the corresponding flavor group [15]. From the point of view of our model-independent description of new scalars, each of these flavor multiplets corresponds to several copies of one of our gauge-covariant multiplets, with correlated couplings. ${ }^{7}$ The presence of tree-level flavor changing neutral currents can also be softened if the new Yukawa interactions are adequately aligned with the SM flavors. In particular, any flavor violation can be removed - up to terms suppressed by the corresponding CKM matrix elements - if, for each scalar multiplet, only one entry of the new Yukawa matrices is non-zero. We will restrict ourselves to this case in the present section. This tuned choice provides conservative bounds. It also helps to establish in which places certain new physics effects might be hidden and to determine their maximum size allowed by current data.

We also noted in the discussion of the previous section that in several cases the new scalars can contribute to other extremely sensitive physical observables, such as proton decay or neutrino masses. Since one can always assign definite $B$ and $L$ numbers to the new scalars, such contributions can only appear as the product of two interactions selecting

\footnotetext{
${ }^{6}$ Besides the obvious simplifications of reducing the number of free parameters and allowing for a simple one-dimensional presentation of the results, this assumption allows us to use the $p p \rightarrow j j$ results of [29]. Indeed, the four-quark operators $\mathcal{O}_{\text {qud }}^{(1)}$ and $\mathcal{O}_{\text {qud }}^{(8)}$, not considered in that reference, are generated for some scalar representations, but their coefficients always involve the product of two different Yukawa couplings.

${ }^{7}$ Since ref. [15] concentrates on quark processes, its classification does not include the scalar representations that do not have purely quark interactions.
} 
different assignments of these quantum numbers. Therefore, they are always avoided when only one of these couplings is non-vanishing.

Finally, certain contributions to four fermion operators that would give rise to chargedcurrent interactions mediating rare decays are also absent when we only consider one nonzero coupling. For instance, the observable $R_{\pi}=\Gamma\left(\pi^{+} \rightarrow \nu e^{+}\right) / \Gamma\left(\pi^{+} \rightarrow \nu \mu^{+}\right)$set bounds on the operators $\mathcal{O}_{q d e}, \mathcal{O}_{\text {ledq }}$ and $\mathcal{O}_{\text {luqe }}$, which are significantly stronger than the ones from the LHC and EWPD considered here [31,32]. The same holds for same-sign top pair production [33].

In the scenario we are considering, with no contribution to any of these sensitive observables, the most relevant constraints on the couplings and masses of the new scalars come from flavor-, $B$ - and $L$-blind observables. Our fits combine the bounds on dimension six interactions from EWPD [34] (see also [35]), ${ }^{8}$ LHC dilepton [32] and dijet searches [29], and measurements of Higgs observables [26]. ${ }^{9}$ In all the analyses we fix the SM inputs to their best-fit values in the absence of extra scalars,

$$
\begin{array}{rlrl}
m_{H} & =125.1 \pm 0.2 \mathrm{GeV}, & & m_{t}=173.8 \pm 0.8 \mathrm{GeV}, \quad M_{Z}=91.1880 \pm 0.0020 \mathrm{GeV}, \\
\alpha_{s}\left(M_{Z}^{2}\right) & =0.1186 \pm 0.0006, & \Delta \alpha_{\text {had }}^{(5)}\left(M_{Z}^{2}\right)=0.02754 \pm 0.00010,
\end{array}
$$

and vary only the new-physics parameters. This is a good approximation, since large effects are not allowed. The limits we obtain in this way are presented in table 2 for the colorless multiplets, and in table 3 for the ones charged under $\mathrm{SU}(3)_{c}$. In all cases the limits apply to ratios of couplings and masses, which are the quantities that appear in the coefficients of the effective operators. (In some cases tailored searches can give better bounds when the new scalars can be directly produced [46].)

Let us comment on the few absences in those tables. In the colorless case, we cannot put meaningful bounds on the quadruplet couplings. As explained in the previous section, they only modify the Higgs self-coupling, which is not significantly constrained by the LHC data at $8 \mathrm{TeV}$. In the colored sector, we have not presented any bounds for $\omega_{2}$, nor for the hadronic couplings of $\omega_{4}$ and $\zeta$. These could be in principle constrained by the LHC dijet data. However, the hadronic couplings of these three multiplets are antisymmetric and necessarily involve more than one family. Hence, they go beyond the first-family approximation used in [29]. Putting bounds on them would require an extended analysis.

Finally, let us discuss the range of validity of the effective Lagrangian. In this approach, the results are given on the ratios $y_{\sigma} / M_{\sigma}$, where $y_{\sigma}<4 \pi$ to allow for a loop expansion, or $\kappa_{\sigma} / M_{\sigma}^{2}$, where we assumed $\left|\kappa_{\sigma}\right| \lesssim M_{\sigma}$. To guarantee the validity of our bounds, we need to assume that the scalar masses are sufficiently larger than the relevant energies and momenta of the processes we consider. This condition depends on each observable and coupling and is always satisfied by large enough values of the masses. But for large masses the upper

\footnotetext{
${ }^{8}$ This includes the usual $Z$-pole data [36], $\Delta \alpha_{\text {had }}^{(5)}\left(M_{Z}^{2}\right)$ [37], $\alpha_{s}\left(M_{Z}^{2}\right)$ [38], the top [39] and Higgs [40, 41] masses, the $W$ mass and width [42, 43], the final LEP2 results of $e^{+} e^{-} \rightarrow \bar{f} f$ [44], unitarity constraints on the the CKM matrix [38], as well as several low-energy measurements [38].

${ }^{9}$ We do not include here limits from (opposite sign) top pair production on couplings mixing the first and third generation of quarks. These can be obtained from LHC data and the results in [45] and [14] and will be considered elsewhere.
} 


\begin{tabular}{|c|c|c|}
\hline Scalar & Parameter & $\begin{array}{c}\text { 95\% C.L. Bound } \\
{\left[\mathrm{TeV}^{-1}\right]}\end{array}$ \\
\hline $\mathcal{S}$ & $\frac{\left|\kappa_{\mathcal{S}}\right|}{M_{\mathcal{S}}^{2}}$ & 1.55 \\
\hline $\mathcal{S}_{1}$ & $\frac{\left|y_{\mathcal{S}_{1}}^{l}\right|}{M_{\mathcal{S}_{1}}}$ & $\left(\begin{array}{ccc}- & 0.08 & - \\
0.08 & - & - \\
- & - & -\end{array}\right)$ \\
\hline $\mathcal{S}_{2}$ & $\frac{\left|y_{\mathcal{S}_{2}}^{e}\right|}{M_{\mathcal{S}_{2}}}$ & $\left(\begin{array}{ccc}0.36 & 0.19 & 0.28 \\
0.19 & - & - \\
0.28 & - & -\end{array}\right)$ \\
\hline$\varphi$ & $\begin{array}{c}\frac{\left|y_{\varphi}^{e}\right|}{M_{\varphi}} \\
\frac{\left|\left(y_{\varphi}^{d}\right)_{11}\right|}{M_{\varphi}} \\
\frac{\left|\left(y_{\varphi}^{u}\right)_{11}\right|}{M_{\varphi}}\end{array}$ & $\begin{array}{c}\left(\begin{array}{ccc}0.26 & 0.56 & 0.79 \\
0.56 & - & - \\
0.79 & - & -\end{array}\right) \\
0.61 \\
0.44\end{array}$ \\
\hline$\Xi_{0}$ & $\frac{\left|\kappa \Xi_{0}\right|}{M_{\Xi_{0}}^{2}}$ & 0.11 \\
\hline$\Xi_{1}$ & $\begin{array}{l}\frac{\left|\kappa \Xi_{1}\right|}{M_{\Xi_{1}}^{2}} \\
\frac{\left|y y_{\Xi_{1}}^{l}\right|}{M \Xi_{1}}\end{array}$ & $\begin{array}{c}0.04 \\
\left(\begin{array}{ccc}0.33 & 0.09 & 0.18 \\
0.09 & - & - \\
0.18 & - & -\end{array}\right)\end{array}$ \\
\hline
\end{tabular}

Table 2. Bounds on the colorless new scalars from flavor-preserving observables. The results for the Yukawa matrices are obtained from a fit to each one of the entries of the coupling matrices at a time. The limit on $\kappa_{\mathcal{S}}$ is determined exclusively by the Higgs data, while the ones on the $\kappa_{\Xi_{i}}$ couplings are dominated by the EWPD limits on the $T$ parameter. Leptonic couplings are constrained by the LEP2 $\left(e^{+} e^{-} \rightarrow \ell^{+} \ell^{-}\right)$and low energy measurements (e.g. Møller and $\nu$-electron scattering), while the hadronic ones are bounded by the LHC dijet angular distributions.

region of the allowed parameters may involve strong couplings that threaten perturbativity and thus the validity of the tree-level approximation. This can happen when the limits on coupling/mass ratios are weak. One example is the scalar singlet $\mathcal{S}$, which is only observable through its contributions to the Higgs boson wave function via the operator $\mathcal{O}_{\phi \square}$, with mild limits $[26,27]$. For $\left|\kappa_{\mathcal{S}}\right| \sim M_{\mathcal{S}}$, the bound in table 2 implies $M_{\mathcal{S}} \gtrsim 700 \mathrm{GeV}$, which is close to the scale probed at the LHC, and the validity of this bound might be questioned. In some cases, entries involving electrons coupling with the second and third family of quarks are also relatively weak $\sim \mathcal{O}(1-2) \mathrm{TeV}^{-1}$. For weakly coupled scenarios $\left(y_{\sigma}<1\right)$ this implies the new scalar masses can be around 500-1000 GeV. However, these entries are only constrained by the LEP 2 data, which involves lower energies $\sqrt{s} \leq 209 \mathrm{GeV}$. Those entries that can modify dilepton production at the LHC can be as large as $\sim 0.5 \mathrm{TeV}^{-1}$ for the case of $\Pi_{1}$. This translates into a mass scale of $\sim 2 \mathrm{TeV}$, which is in principle 


\begin{tabular}{|c|c|c|}
\hline Scalar & Parameter & $\begin{array}{c}95 \% \text { C.L. Bound } \\
{\left[\mathrm{TeV}^{-1}\right]}\end{array}$ \\
\hline$\omega_{1}$ & $\begin{array}{c}\frac{\left|y_{\omega_{1}}^{q l}\right|}{M_{\omega_{1}}} \\
\frac{\left|\left(y_{\omega_{1}}^{q q}\right)_{11}\right|}{M_{\omega_{1}}} \\
\frac{\left|y_{\omega_{1}}^{e u}\right|}{M_{\omega_{1}}} \\
\frac{\left|\left(y_{\omega_{1}}^{d u}\right)_{11}\right|}{M_{\omega_{1}}}\end{array}$ & $\begin{array}{c}\left(\begin{array}{ccc}0.19 & 0.53 & - \\
0.40 & - & - \\
- & - & -\end{array}\right) \\
0.24 \\
\left(\begin{array}{ccc}0.27 & 0.49 & - \\
0.48 & - & - \\
- & - & -\end{array}\right) \\
0.47\end{array}$ \\
\hline$\omega_{4}$ & $\frac{\left|y_{\omega_{4}}^{e d}\right|}{M_{\omega_{4}}}$ & $\left.\begin{array}{ccc}0.28 & 0.98 & 0.98 \\
0.42 & - & - \\
- & - & -\end{array}\right)$ \\
\hline$\Pi_{1}$ & $\frac{y_{\Pi_{1}} \mid}{M_{\Pi_{1}}}$ & $\left.\begin{array}{ccc}0.27 & 1.80 & 1.80 \\
0.48 & - & - \\
- & - & -\end{array}\right)$ \\
\hline
\end{tabular}

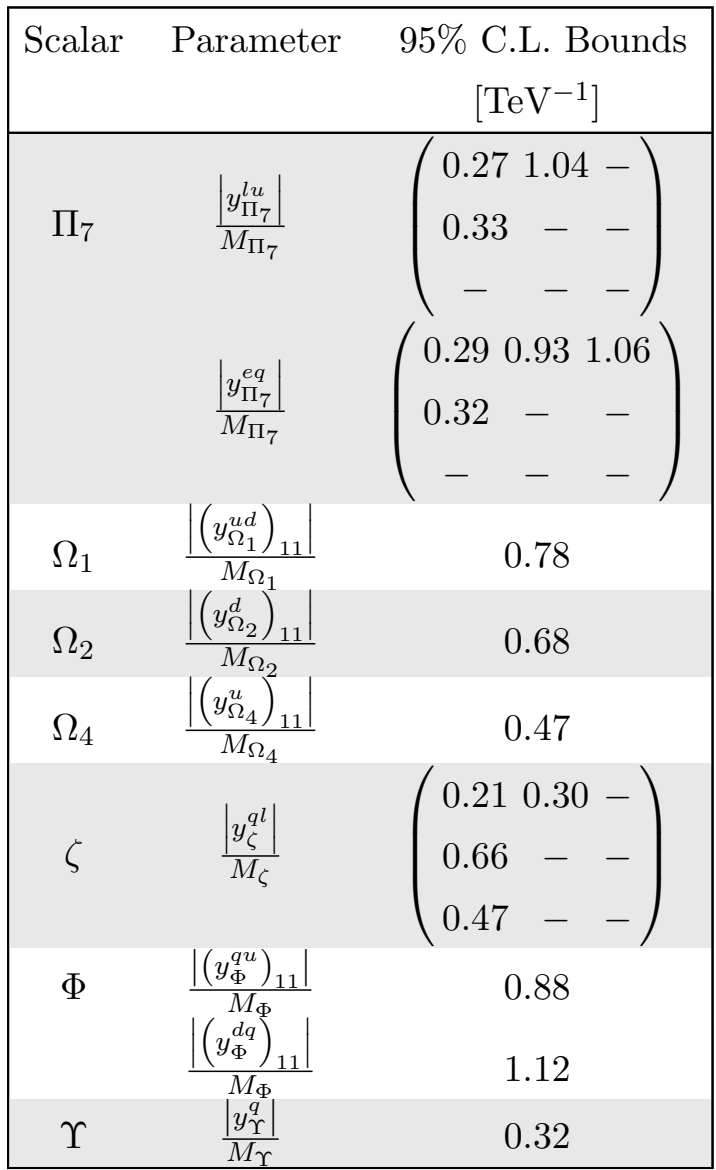

Table 3. Bounds on the colored new scalars from flavor-preserving observables. The results for the Yukawa matrices are obtained from a fit to each one of the entries of the coupling matrices at a time. All these interactions are constrained by two to two fermion processes. Leptoquark interactions are bounded by LEP $2 e^{+} e^{-} \rightarrow$ had data, low energy measurements (e.g. Atomic parity violation, $\nu$-nucleon scattering), CKM unitarity, and dilepton searches at the LHC. Purely hadronic bounds are again obtained only from the LHC $p p \rightarrow j j$ angular distributions. See text for more details.

accessible by the LHC at $\sqrt{s}=8 \mathrm{TeV}$. However, dilepton processes at the LHC can only be mediated by leptoquarks in $t$-channel. Thus, even for such values of masses and weak couplings, the effective theory remains valid in a large region of the phase space and can give a good approximation to the integrated observables (see the quantitative discussion of an analogous process in [14]). Finally, the results for the pure hadronic interactions should be taken with care. Again, for order one couplings the corresponding mass scales can be relatively small, while the dijet angular distributions used in [29] to set bounds correspond to dijet masses $M_{j j}>3 \mathrm{TeV}$. Unlike the dilepton case, these scalars can be produced in $s$-channel. For instance, for the case with the weakest bound, the color-octet iso-doublet $\Phi$, demanding $M_{\Phi}>3.9 \mathrm{TeV}$ ( the highest $M_{j j}$ value observed in the CMS analysis [47] used in [29]), the hadronic couplings needed to saturate the bounds must be $\left(y_{\Phi}^{d q}\right)_{11} \gtrsim 4$, and one may start worrying about the precision of the perturbative (asymptotic) series. Note, nevertheless, that for $s$-channel processes the limits obtained with the effective Lagrangian give a conservative estimate of the actual limit. In summary, with the current constraints 
the effective Lagrangian approach provides in general a good approximation for heavy scalars and a large range of values of their couplings.

\section{Scalar extensions with other new particles}

In the previous section we have considered in detail SM extensions with only one scalar multiplet. In this case, there are strong correlations between different observables. In this section we discuss the interplay between the effects of different scalars, and also between particles with different spin. The effective Lagrangian formalism used in this article allows an easy comparison of the effects of different sources of new physics. In particular, it helps to identify at the Lagrangian level those places where a (partial) cancellation between the virtual effects of different new particles in physical observables can take place [48, 49]. This is useful for model builders to construct scenarios with not too heavy (or not too weakly coupled) particles that are consistent with the existing phenomenological constraints. Such cancellations require a large correlation between the effects of different new particles. Although they correspond to small regions in the parameter space of generic models, in some cases they can be made natural by imposing extra symmetries.

Let us first point out that, at the tree level, the dimension-six effective Lagrangian in extensions of the SM with arbitrary new particles of spin $0,1 / 2$ and 1 is simply the sum of the effective Lagrangian obtained here and the ones in refs. [5-7]. ${ }^{10}$ Indeed, a simple extension of the argument in section 3 shows that mixed contributions from particles of different spin only appear at higher dimension. Therefore, the effective Lagrangians in those references and in this paper completely characterize the largest effects of arbitrary extensions of the SM with new heavy particles. Note in this regard that particles of spin higher than 1 only interact via non-renormalizable couplings, which are naturally suppressed.

In what follows we study, for each type of interaction induced by the extra scalars to dimension six, the different sources of new physics (new scalars, fermions or vectors) that can cancel at the tree-level the effects from the virtual exchange of scalar bosons. We discuss the cancellations at the operator level, which is a sufficient (and often necessary) condition to guarantee the cancellation in physical observables.

- Dimension-five operators: the Weinberg operator only arises when we integrate the hypercharge-one iso-triplet $\Xi_{1}$. Having no definite sign, contributions from different triplets could cancel each other, or the ones coming from the other two possible seesaw messengers, i.e. new lepton singlets and triplets with hypercharge zero.

- Oblique operators: cancellation between custodial isospin breaking contributions can occur between the two triplets, as both have definite, opposite sign. This is also

\footnotetext{
${ }^{10} \mathrm{~A}$ direct comparison of the effective Lagrangian results presented here with those in refs. [5-7] requires to perform certain field redefinitions and Fierz reorderings, since the basis employed in those works has some redundant interactions, and use different definitions for some operators. All the transformations needed to relate both bases are provided in appendix A, where we also introduce the full basis of dimension six operators we use, which can be compared to the one in table 7 in ref. [7].
} 
possible with new vector singlets with hypercharge zero (hypercharge one), which yield negative (positive) definite contributions to $\alpha_{\phi D}$. New vector triplets with hypercharge one also yield negative contributions to this operator [7].

- Scalar operators: these include $\mathcal{O}_{\phi}$ and $\mathcal{O}_{\phi \square}$. A look at the second row of table 28 in appendix B shows all the possible contributions to the operator $\mathcal{O}_{\phi}$, including collective contributions that appear when two different species of new scalars are present at the same time. Contributions to $\mathcal{O}_{\phi \square}$, on the other hand, are much simpler, and are negative (positive) definite for $\mathcal{S}\left(\Xi_{0,1}\right)$. This allows for cancellations between colorless iso-singlets and iso-triplets.

- Scalar-Fermion operators: in extensions with new scalars only, these operators only appear in the case of colorless iso-doublets, $\varphi$ or iso-triplets, $\Xi_{0,1}$. However, for the latter they always arise through a field redefinition, necessary to bring all the contributions in the dimension-six Lagrangian into the chosen basis. As a consequence, the flavor structure of the coefficients of the operators $\mathcal{O}_{e \phi}$ and $\mathcal{O}_{d \phi}$, and $\mathcal{O}_{u \phi}$ coming from triplets is always SM-like (proportional to the SM Yukawa couplings), while the one from doublets $\varphi$ can be completely generic. Moreover, while all the contributions of arbitrary triplets have the same sign, the sign of the genuine contributions from $\varphi$ is indefinite. Therefore, cancellations between scalars are always possible.

Contributions from new vectors [7], also appear when the SM equations of motion are used. They only come from colorless hypercharge zero or one iso-singlets, or iso-triplets $\left(\mathcal{B}, \mathcal{B}_{1}, \mathcal{W}\right.$ and $\mathcal{W}_{1}$ in the notation of [7]), and are also SM-like.

Finally, heavy fermions can generate these contributions either after applying the SM equation of motion, if only one fermion species is present, or as a result of the combined effect of extra fermionic iso-doublets and new fermion iso-singlets or iso-triplets $[5,6]$. Even in the case of only one fermion, the contributions to $\mathcal{O}_{f \phi}$ always involve the flavor structure of the new fermionic interactions, and therefore are general a priori. Hence, some interplay with the contributions from scalar doublets is possible, although an eventual cancellation of all the scalar effects may require several different new fermion multiplets.

- Four-fermion operators: upon inspection of the new scalar contributions to fourfermion operators, it can be seen that, for a fixed set of flavor indices with $i=j, k=l$, the operator coefficients of all four-fermion interactions involving at most two different types of SM fermion multiplets have a definite sign. Although the contributions to four-fermion interactions with three or four different multiplets have no definite sign, they are always correlated with operators involving only one or two kinds of multiplets. Moreover, for a particular operator, contributions from scalars of different types have either the same sign, or are proportional to the contribution to another operator with the same field content where both scalars contribute additively. For instance, $\varphi$ and $\Phi$ contributions to $\alpha_{q u}^{(8)}$ have opposite sign and can balance each other. However, each individual contribution is proportional to the corresponding one to $\alpha_{q u}^{(1)}$, where both have the same sign. Therefore, a complete cancellation 
of the effects from such couplings to two-to-two fermion processes is not possible in extensions with extra scalars only. This is quite similar to the situation for extra vectors [7]. However, as illustrated in ref. [49] for the case of pure leptonic interactions, a cancellation between the four fermion effects coming from new scalars and the ones from new vector particles is possible in many cases, although it comes at the price of a significant fine tuning. New vectors of hypercharge $Y \neq 0$ contribute with a definite sign to four-fermion operators involving at most two types of SM multiplets if $i=j, k=l$, exactly as in the scalar case. For vectors of zero hypercharge, only the operators where all fermions belong to the same SM representation can have a definite sign. This is always the case for $i=j=k=l$ and, in certain cases, for $i=l, j=k .^{11}$ Instead of going over each operator/scalar/vector and listing all the possible cancellations, we show in table 4 those interactions that are common for each scalar-vector pair, indicating the relative sign between the different contributions for both cases, $i=l, j=k(i \neq j)$, and $i=j=k=l$ (where some restrictions appear in the case of hypercharge-zero vector fields). In general, for a given operator with four multiplets of the same kind, one can always choose a scalar and a hypercharge-zero vector field such that, tuning the corresponding scalar/vector couplings, contributions with opposite sign are obtained. Table 5 contains the same information for the case of four-fermion operators built from at most two types of multiplets, with $i=j, k=l(i \neq k)$.

Note that a relative minus sign between the contributions from two particles to a given operator does not always guarantee that a complete cancellation of the new physics effects is possible. The reason is that the contributions to some operators with the same field content are in many cases correlated, and a cancellation in all those operator coefficients does not usually take place at the same time. However, for each configuration of four fermionic fields, there are at most two independent operators in the dimension six basis. And, as can be seen from the tables, for each scalar (vector) field and pair of such operators, one can always find a pair of vectors (scalars) that contribute to both operators with the adequate signs to cancel the total contribution. Therefore, we conclude that for any given four-fermion process receiving contributions from one new particle through an arbitrary set of four-fermion operators, it is always possible to find a combination of new fields that, after the adequate tuning in their couplings, cancels out all the new effects. Correlations with other types of operators can be easily avoided.

Summarizing, we see that the existing experimental limits are compatible with many combinations of new particles with sizable couplings and masses at the LHC reach. Essentially, by including many new multiplets we are breaking the correlations in the coefficients of the effective operators. However, in most cases the corresponding models are too contrived and fine tuned. In the simplest cases, symmetries may exist which make these models more natural and appealing. The discussion in this section may be useful in the search of such symmetries.

\footnotetext{
${ }^{11}$ Note that in this case, the corresponding scalar contributions always have a definite sign.
} 


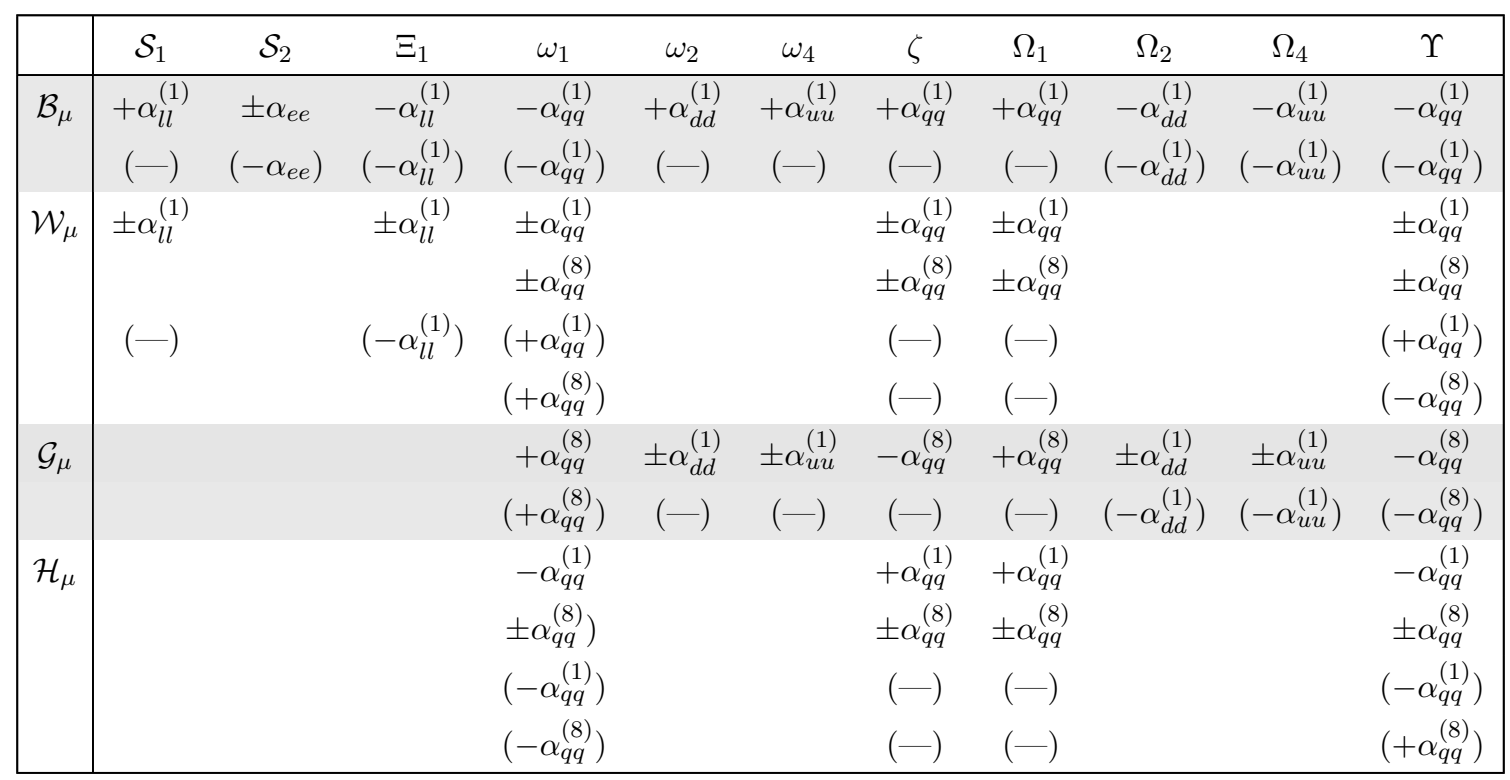

Table 4. Contributions to four-fermion interactions with $i=l, j=k(i \neq j)$, common to new scalar and (hypercharge-zero) vector fields. Only operators involving one type of multiplet, and the particles that contribute to them, are shown. The symbols "+" ("-") indicate that the contributions from scalars and vectors have the same (opposite) sign, while " \pm " indicates the absence of a definite sign in any of the sources. The same information for the case $i=j=k=l$ is provided in parenthesis. In this case, a dash ("-") indicates the absence of contribution from the corresponding scalar particle.

\section{Conclusions}

The discovery at the LHC of a new particle of spin 0 has come hand in hand with the direct observation of new interactions mediated by scalar fields. ${ }^{12}$ Among these, the Yukawa interactions are quite unique in that they are not ruled by gauge invariance under the SM gauge group, although of course they are compatible with it. The exploration of this scalar sector is an important part of the LHC physics program. The results at the LHC Run 1 have already constrained significantly its structure. So far, all the measurements are consistent with the minimal scalar sector of the SM: a Higgs iso-doublet with a non-vanishing vev for its neutral component. But the present uncertainties still allow for significant deviations in the couplings of this doublet and for the presence of additional scalar fields, related or not to electroweak symmetry breaking. To comply with all the available data, such extra scalars must either have small couplings or be significantly heavier than the Higgs boson. The latter is the scenario we have considered in the second part of this paper.

We have followed a largely model-independent and unbiased approach, with the minimal theoretical input of gauge-invariance. First, we have classified into 19 irreducible representations all the possible scalar fields that can interact linearly with the SM fields with gauge-invariant renormalizable couplings. Their components with a definite electric charge are the only scalar particles that can be produced at colliders with unsuppressed

\footnotetext{
${ }^{12}$ Indirect evidence of gauge-Higgs interactions was available before, in EWPD.
} 


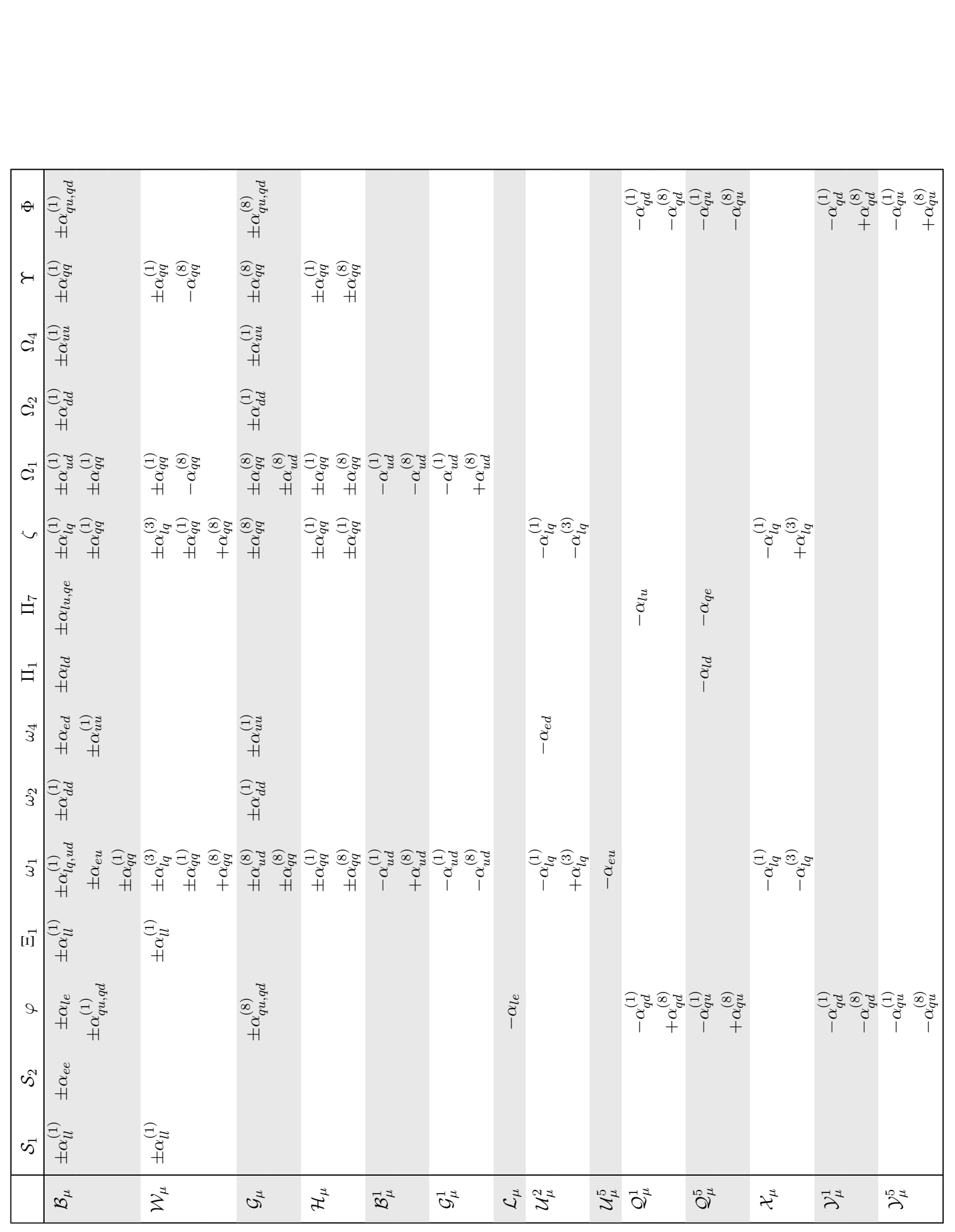

总产焉

용

范

要

苛

500

$\overrightarrow{0}$

总

营

蛋

ठ 마

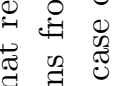

$\exists$ ॠ

丞

迅

वे :

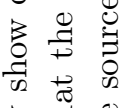

궘

○ 荧

उ.尹

घ: $\exists . \exists$

可 $\approx$

ㄷำ

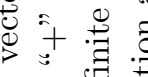

경 융 㝵

के है

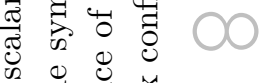

क

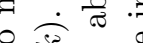

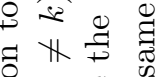

00 is

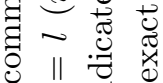

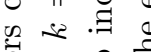

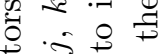

要

青它品

莺 总

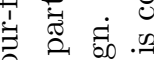

诖

10.

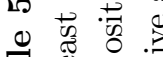

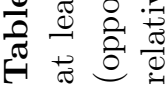


couplings. We have written the most general renormalizable interactions of the scalar multiplets (except for parts of the scalar potential that cannot be tested in the near future). Up to this point, all our results apply to either light or heavy extra scalars. In a second step, we have assumed a hierarchy of scales and have derived the dimension-six effective Lagrangian that describes all the tree-level effects of the heavy scalars in experiments where the probed energies are smaller than their masses. We have shown that only the 19 scalar multiplets with allowed linear interactions contribute to operators of dimension five and six. Non-linear interactions of these fields also appear in the effective Lagrangian to this order. The results are collected in appendix B. Finally, we have used this effective Lagrangian to discuss the observable effects of the new scalars and to derive bounds on their couplings and masses. The strongest bounds come from flavor observables. In order to avoid flavor constraints, here we have simply assumed that, in the flavor basis defined by eq. (2.2), there is only one non-vanishing entry in the Yukawa couplings with the new scalar. We have then studied the limits from a range of flavor-conserving observables: EWPD, LHC dilepton and dijet searches and Higgs-mediated cross sections.

Together with refs. [5-7], this paper provides a complete classification of all the particles with up to dimension-four linear couplings (in the electroweak symmetric phase) to the SM fields. Even if our emphasis in this paper has been on indirect effects, let us stress that this classification and the general interactions that are explicitly written in these references provide a useful basis for model-independent direct searches at large colliders (see e.g. $[12,50-56]$ for applications of this gauge-invariant formalism to direct searches).

On top of this, our results here complete the tree-level dictionary between particles with general couplings of dimension $\leq 4$ and the effective operators that describe their lowenergy effects. The dictionary entries for quarks, leptons and vector bosons can be found in refs. [5-7], respectively. We believe this correspondence can prove useful in combining the information from LHC searches of new particles with the existing precision constraints on their masses and couplings.

\section{Acknowledgments}

We thank L. Silvestrini and A. Weiler for useful discussions. The work of JB has been supported by the European Research Council under the European Union's Seventh Framework Programme (FP/2007-2013)/ERC Grant Agreement n. 279972. The work of MC, MPV and JS has been partially supported by the European Commission through the contract PITNGA-2012-316704 (HIGGSTOOLS), by MINECO, under grant numbers FPA2010-17915 and FPA2013-47836-C3-2-P, and by the Junta de Andalucía grants FQM 101 and FQM 6552.

\section{A Basis of dimension-six operators}

In this appendix, we introduce a complete set of gauge-invariant operators $\mathcal{O}_{i}$, which enter the general SM effective Lagrangian to dimension six:

$$
\mathcal{L}_{\mathrm{Eff}}^{(6)}=\mathcal{L}_{\mathrm{SM}}+\frac{1}{\Lambda} \mathcal{L}_{5}+\frac{1}{\Lambda^{2}} \mathcal{L}_{6}, \quad \text { with } \quad \mathcal{L}_{d}=\sum_{i} \alpha_{i} \mathcal{O}_{i}
$$




\begin{tabular}{|c|c|c|c|c|}
\hline & Operator & Notation & Operator & Notation \\
\hline \multirow{3}{*}{$\begin{array}{l}\not \\
\text { ä } \\
\ddot{a}\end{array}$} & $\left(\phi^{\dagger} \phi\right)^{2}$ & $\mathcal{O}_{\phi 4}$ & $\overline{l_{L}} \phi e_{R}$ & $\mathcal{O}_{y_{e}}$ \\
\hline & & & $\overline{q_{L}} \tilde{\phi} u_{R}$ & $\mathcal{O}_{y_{u}}$ \\
\hline & & & $\overline{q_{L}} \phi d_{R}$ & $\mathcal{O}_{y_{d}}$ \\
\hline 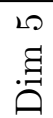 & $\overline{\overline{l_{L}^{c}}} \tilde{\phi}^{*} \tilde{\phi}^{\dagger} l_{L}$ & $\mathcal{O}_{5}$ & & \\
\hline
\end{tabular}

Table 6. Operators of dimension four and five.

We employ the basis in tables 6,7 and 8 . Table 6 defines our notation for the effective operators renormalizing the SM interactions, and presents the unique dimension-five interaction: the Weinberg operator, which gives Majorana masses to the SM neutrinos. Tables 7 and 8 gather the dimension-six operators. In these tables, $T_{A}=\frac{1}{2} \lambda_{A}$ and $f_{A B C}$, $A, B, C=1, \ldots, 8$, are the $\mathrm{SU}(3)_{c}$ generators and structure constants, with $\lambda_{A}$ the GellMann matrices; $\epsilon_{A B C}\left(\varepsilon_{a b c}\right), A, B, C=1,2,3(a, b, c=1,2,3)$ is the totally antisymmetric tensor in color (weak isospin) indices; $\sigma_{a}, a=1,2,3$ are the Pauli matrices; and $\widetilde{A}_{\mu \nu}=\frac{1}{2} \varepsilon_{\mu \nu \rho \sigma} A^{\rho \sigma}$ is the Hodge-dual of the field strength $A_{\mu \nu}$. Finally, the superscript symbol "T" denotes transposition of the $\mathrm{SU}(2)_{L}$ indices exclusively.

We use essentially the same basis as the one introduced in ref. [57]. (See [58-62] for a related discussion of dimension-six physics in different operator bases.) The only differences (apart from changes in the names) are the use of different normalization factors in several operators, and the trade of their operators $Q_{q q}^{(3)}=\left(\overline{q_{L}} \gamma_{\mu} \sigma_{a} q_{L}\right)\left(\overline{q_{L}} \gamma^{\mu} \sigma_{a} q_{L}\right)$ and $Q_{\text {lequ }}^{(3)}=$ $\left(\overline{l_{L}} \sigma_{\mu \nu} e_{R}\right) i \sigma_{2}\left(\overline{q_{L}} \sigma^{\mu \nu} u_{R}\right)^{\mathrm{T}}$ by $\mathcal{O}_{q q}^{(8)}$ and $\mathcal{O}_{\text {luqe }}$, respectively, in our tables. Also, for consistency with previous works we write here the operators $\mathcal{O}_{\phi \psi}^{(1)}=\left(\phi^{\dagger} i D_{\mu} \phi\right)\left(\bar{\psi} \gamma^{\mu} \psi\right)$ and $\mathcal{O}_{\phi \psi}^{(3)}=$ $\left(\phi^{\dagger} i \sigma_{a} D_{\mu} \phi\right)\left(\overline{\psi_{L}} \gamma^{\mu} \sigma_{a} \psi_{L}\right)$, instead of the hermitian interactions $\mathcal{Q}_{\phi \psi}^{(1)}=\left(\phi^{\dagger} i \stackrel{\leftrightarrow}{D_{\mu}} \phi\right)\left(\bar{\psi} \gamma^{\mu} \psi\right)$ and $\mathcal{Q}_{\phi \psi}^{(1)}=\left(\phi^{\dagger} i \stackrel{\leftrightarrow}{D_{\mu}^{a} \phi}\right)\left(\overline{\psi_{L}} \gamma^{\mu} \sigma_{a} \psi_{L}\right)$ of ref. [57]. Note that these last interactions are not generated in the integration of the new scalars, and are introduced here only for completeness.

Finally, for the purpose of comparing the results of the integration of new scalars with those obtained for new fermions and vector bosons in refs. [5-7], we provide below the necessary relations to translate the results in those references, which use the original basis of $[58,59]$ and therefore contains redundant interactions, into our basis. Again, we use the notation $\mathcal{Q}_{i}$ to refer to the operator basis in other references, while we keep $\mathcal{O}_{i}$ for the operators presented in tables 7 and 8 .

In the sector of four-fermion interactions the following identities follow from the corresponding Fierz reorderings:

$$
\begin{aligned}
\left(\mathcal{Q}_{l l}^{(3)}\right)_{i j k l} & =\frac{1}{2}\left(\overline{l_{L}^{i}} \gamma_{\mu} \sigma_{a} l_{L}^{j}\right)\left(\overline{l_{L}^{k}} \gamma^{\mu} \sigma_{a} l_{L}^{l}\right)=2\left(\mathcal{O}_{l l}^{(1)}\right)_{i l k j}-\left(\mathcal{O}_{l l}^{(1)}\right)_{i j k l}, \\
\left(\mathcal{Q}_{q q}^{(1,3)}\right)_{i j k l} & =\frac{1}{2}\left(\overline{q_{L}^{i}} \gamma_{\mu} \sigma_{a} q_{L}^{j}\right)\left(\overline{q_{L}^{k}} \gamma^{\mu} \sigma_{a} q_{L}^{l}\right)=-\left(\mathcal{O}_{q q}^{(1)}\right)_{i j k l}+\frac{2}{3}\left(\mathcal{O}_{q q}^{(1)}\right)_{i l k j}+4\left(\mathcal{O}_{q q}^{(8)}\right)_{i l k j},
\end{aligned}
$$




\begin{tabular}{|c|c|c|c|c|}
\hline & Operator & Notation & Operator & Notation \\
\hline \multirow{3}{*}{ 兒 } & $\frac{1}{2}\left(\overline{l_{L}} \gamma_{\mu} l_{L}\right)\left(\overline{l_{L}} \gamma^{\mu} l_{L}\right)$ & $\mathcal{O}_{l l}^{(1)}$ & & \\
\hline & $\frac{1}{2}\left(\overline{q_{L}} \gamma_{\mu} q_{L}\right)\left(\overline{q_{L}} \gamma^{\mu} q_{L}\right)$ & $\mathcal{O}_{q q}^{(1)}$ & $\frac{1}{2}\left(\overline{q_{L}} \gamma_{\mu} T_{A} q_{L}\right)\left(\overline{q_{L}} \gamma^{\mu} T_{A} q_{L}\right)$ & $\mathcal{O}_{q q}^{(8)}$ \\
\hline & $\left(\overline{l_{L}} \gamma_{\mu} l_{L}\right)\left(\overline{q_{L}} \gamma^{\mu} q_{L}\right)$ & $\mathcal{O}_{l q}^{(1)}$ & $\left(\overline{l_{L}} \gamma_{\mu} \sigma_{a} l_{L}\right)\left(\overline{q_{L}} \gamma^{\mu} \sigma_{a} q_{L}\right)$ & $\mathcal{O}_{l q}^{(3)}$ \\
\hline \multirow{4}{*}{ 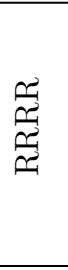 } & $\frac{1}{2}\left(\overline{e_{R}} \gamma_{\mu} e_{R}\right)\left(\overline{e_{R}} \gamma^{\mu} e_{R}\right)$ & $\mathcal{O}_{e e}$ & & \\
\hline & $\frac{1}{2}\left(\overline{u_{R}} \gamma_{\mu} u_{R}\right)\left(\overline{u_{R}} \gamma^{\mu} u_{R}\right)$ & $\mathcal{O}_{u u}^{(1)}$ & $\frac{1}{2}\left(\overline{d_{R}} \gamma_{\mu} d_{R}\right)\left(\overline{d_{R}} \gamma^{\mu} d_{R}\right)$ & $\mathcal{O}_{d d}^{(1)}$ \\
\hline & $\left(\overline{u_{R}} \gamma_{\mu} u_{R}\right)\left(\overline{d_{R}} \gamma^{\mu} d_{R}\right)$ & $\mathcal{O}_{u d}^{(1)}$ & $\left(\overline{u_{R}} \gamma_{\mu} T_{A} u_{R}\right)\left(\overline{d_{R}} \gamma^{\mu} T_{A} d_{R}\right)$ & $\mathcal{O}_{u d}^{(8)}$ \\
\hline & $\left(\overline{e_{R}} \gamma_{\mu} e_{R}\right)\left(\overline{u_{R}} \gamma^{\mu} u_{R}\right)$ & $\mathcal{O}_{e u}$ & $\left(\overline{e_{R}} \gamma_{\mu} e_{R}\right)\left(\overline{d_{R}} \gamma^{\mu} d_{R}\right)$ & $\mathcal{O}_{e d}$ \\
\hline \multirow{5}{*}{ 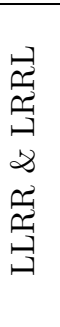 } & $\left(\overline{l_{L}} \gamma_{\mu} l_{L}\right)\left(\overline{e_{R}} \gamma^{\mu} e_{R}\right)$ & $\mathcal{O}_{l e}$ & $\left(\overline{q_{L}} \gamma_{\mu} q_{L}\right)\left(\overline{e_{R}} \gamma^{\mu} e_{R}\right)$ & $\mathcal{O}_{q e}$ \\
\hline & $\left(\overline{l_{L}} \gamma_{\mu} l_{L}\right)\left(\overline{u_{R}} \gamma^{\mu} u_{R}\right)$ & $\mathcal{O}_{l u}$ & $\left(\overline{l_{L}} \gamma_{\mu} l_{L}\right)\left(\overline{d_{R}} \gamma^{\mu} d_{R}\right)$ & $\mathcal{O}_{l d}$ \\
\hline & $\left(\overline{q_{L}} \gamma_{\mu} q_{L}\right)\left(\overline{u_{R}} \gamma^{\mu} u_{R}\right)$ & $\mathcal{O}_{q u}^{(1)}$ & $\left(\overline{q_{L}} \gamma_{\mu} T_{A} q_{L}\right)\left(\overline{u_{R}} \gamma^{\mu} T_{A} u_{R}\right)$ & $\mathcal{O}_{q u}^{(8)}$ \\
\hline & $\left(\overline{q_{L}} \gamma_{\mu} q_{L}\right)\left(\overline{d_{R}} \gamma^{\mu} d_{R}\right)$ & $\mathcal{O}_{q d}^{(1)}$ & $\left(\overline{q_{L}} \gamma_{\mu} T_{A} q_{L}\right)\left(\overline{d_{R}} \gamma^{\mu} T_{A} d_{R}\right)$ & $\mathcal{O}_{q d}^{(8)}$ \\
\hline & $\left(\overline{l_{L}} e_{R}\right)\left(\overline{d_{R}} q_{L}\right)$ & $\mathcal{O}_{l e d q}$ & & \\
\hline \multirow{2}{*}{ 㟧 } & $\left(\overline{q_{L}} u_{R}\right) i \sigma_{2}\left(\overline{q_{L}} d_{R}\right)^{\mathrm{T}}$ & $\mathcal{O}_{q u d}^{(1)}$ & $\left(\overline{q_{L}} T_{A} u_{R}\right) i \sigma_{2}\left(\overline{q_{L}} T_{A} d_{R}\right)^{\mathrm{T}}$ & $\mathcal{O}_{q u d}^{(8)}$ \\
\hline & $\left(\overline{l_{L}} e_{R}\right) i \sigma_{2}\left(\overline{q_{L}} u_{R}\right)^{\mathrm{T}}$ & $\mathcal{O}_{l e q u}$ & $\left(\overline{l_{L}} u_{R}\right) i \sigma_{2}\left(\overline{q_{L}} e_{R}\right)^{\mathrm{T}}$ & $\mathcal{O}_{\text {luqe }}$ \\
\hline \multirow{3}{*}{$\begin{array}{l}\lambda \\
\infty \\
\not\end{array}$} & $\epsilon_{A B C}\left(\overline{l_{L}} i \sigma_{2} q_{L}^{c}{ }^{A}\right)\left(\overline{d_{R}^{B}} u_{R}^{c}{ }^{C}\right)$ & $\mathcal{O}_{l q d u}$ & $\epsilon_{A B C}\left(\overline{q_{L}^{A}} i \sigma_{2} q_{L}^{c}{ }^{B}\right)\left(\overline{e_{R}} u_{R}^{c}{ }^{C}\right)$ & $\mathcal{O}_{q q e u}$ \\
\hline & $\epsilon_{A B C}\left(\overline{l_{L}} i \sigma_{2} q_{L}^{c}{ }^{A}\right)\left(\overline{q_{L}^{B}} i \sigma_{2} q_{L}^{c}{ }^{C}\right)$ & $\mathcal{O}_{l q q q}^{(1)}$ & $\left.\epsilon_{A B C}\left(\overline{u_{R}^{A}} d_{R}^{c B}\right)\left(\overline{e_{R}} u_{R}^{c}\right)^{C}\right)$ & $\mathcal{O}_{u d e u}$ \\
\hline & $\epsilon_{A B C}\left(\overline{l_{L}} \sigma_{a} i \sigma_{2} q_{L}^{c}{ }^{A}\right)\left(\overline{q_{L}^{B}} \sigma_{a} i \sigma_{2} q_{L}^{c}{ }^{C}\right)$ & $\mathcal{O}_{l q q q}^{(3)}$ & & \\
\hline
\end{tabular}

Table 7. Basis of dimension-six operators used in our analysis: four-fermion contact interactions. Flavor indices are omitted.

$$
\begin{aligned}
\left(\mathcal{Q}_{q q}^{(8,1)}\right)_{i j k l} & =\frac{1}{2}\left(\overline{q_{L}^{i}} \gamma_{\mu} \lambda_{A} q_{L}^{j}\right)\left(\overline{q_{L}^{k}} \gamma^{\mu} \lambda_{A} q_{L}^{l}\right)=4\left(\mathcal{O}_{q q}^{(8)}\right)_{i j k l}, \\
\left(\mathcal{Q}_{q q}^{(8,3)}\right)_{i j k l} & =\frac{1}{2}\left(\overline{q_{L}^{i}} \gamma_{\mu} \lambda_{A} \sigma_{a} q_{L}^{j}\right)\left(\overline{q_{L}^{k}} \gamma^{\mu} \lambda_{A} \sigma_{a} q_{L}^{l}\right)=\frac{32}{9}\left(\mathcal{O}_{q q}^{(1)}\right)_{i l k j}-4\left(\mathcal{O}_{q q}^{(8)}\right)_{i j k l}-\frac{8}{3}\left(\mathcal{O}_{q q}^{(8)}\right)_{i l k j}, \\
\left(\mathcal{Q}_{f f}^{(8)}\right)_{i j k l} & =\frac{1}{2}\left(\overline{f_{R}^{i}} \gamma_{\mu} \lambda_{A} f_{R}^{j}\right)\left(\overline{f_{R}^{k}} \gamma^{\mu} \lambda_{A} f_{R}^{l}\right)=2\left(\mathcal{O}_{f f}^{(1)}\right)_{i l k j}-\frac{2}{3}\left(\mathcal{O}_{f f}^{(1)}\right)_{i j k l}, \quad(f=u, d) \\
\left(\mathcal{Q}_{F f}\right)_{i j k l} & =\left(\overline{F_{L}^{i}} f_{R}^{j}\right)\left(\overline{f_{R}^{k}} F_{L}^{l}\right)=-\frac{1}{2}\left(\mathcal{O}_{F f}\right)_{i l k j}, \quad(F f=l e, l u, l d, q e) \\
\left(\mathcal{Q}_{q f}^{(1)}\right)_{i j k l} & =\left(\overline{q_{L}^{i}} f_{R}^{j}\right)\left(\overline{f_{R}^{k}} q_{L}^{l}\right)=-\frac{1}{6}\left(\mathcal{O}_{q f}^{(1)}\right)_{i l k j}-\left(\mathcal{O}_{q f}^{(8)}\right)_{i l k j}, \quad(f=u, d) \\
\left(\mathcal{Q}_{q f}^{(8)}\right)_{i j k l} & =\left(\overline{q_{L}^{i}} \lambda_{A} f_{R}^{j}\right)\left(\overline{f_{R}^{k}} \lambda_{A} q_{L}^{l}\right)=-\frac{8}{9}\left(\mathcal{O}_{q f}^{(1)}\right)_{i l k j}+\frac{2}{3}\left(\mathcal{O}_{q f}^{(8)}\right)_{i l k j} \cdot
\end{aligned}
$$

Finally, the operator $\mathcal{Q}_{q d e}$ is labeled as $\mathcal{O}_{\text {ledq }}$ in table 7 .

There are also some differences in the case of the bosonic operators. Firstly, the operators $\mathcal{Q}_{\phi 6}$ and $\mathcal{Q}_{\phi}^{(3)}$ correspond exactly to $\mathcal{O}_{\phi D}$ and $\mathcal{O}_{\phi}$, respectively. Secondly, using 


\begin{tabular}{|c|c|c|c|c|}
\hline & Operator & Notation & Operator & Notation \\
\hline en & $\left(\phi^{\dagger} \phi\right) \square\left(\phi^{\dagger} \phi\right)$ & $\mathcal{O}_{\phi \square}$ & $\frac{1}{3}\left(\phi^{\dagger} \phi\right)^{3}$ & $\mathcal{O}_{\phi}$ \\
\hline \multirow{5}{*}{$\sum_{\infty}^{1}$} & $\left(\phi^{\dagger} i D_{\mu} \phi\right)\left(\overline{l_{L}} \gamma^{\mu} l_{L}\right)$ & $\mathcal{O}_{\phi l}^{(1)}$ & $\left(\phi^{\dagger} \sigma_{a} i D_{\mu} \phi\right)\left(\overline{l_{L}} \gamma^{\mu} \sigma_{a} l_{L}\right)$ & $\mathcal{O}_{\phi l}^{(3)}$ \\
\hline & $\left(\phi^{\dagger} i D_{\mu} \phi\right)\left(\overline{e_{R}} \gamma^{\mu} e_{R}\right)$ & $\mathcal{O}_{\phi e}^{(1)}$ & & \\
\hline & $\left(\phi^{\dagger} i D_{\mu} \phi\right)\left(\overline{q_{L}} \gamma^{\mu} q_{L}\right)$ & $\mathcal{O}_{\phi q}^{(1)}$ & $\left(\phi^{\dagger} \sigma_{a} i D_{\mu} \phi\right)\left(\overline{q_{L}} \gamma^{\mu} \sigma_{a} q_{L}\right)$ & $\mathcal{O}_{\phi q}^{(3)}$ \\
\hline & $\left(\phi^{\dagger} i D_{\mu} \phi\right)\left(\overline{u_{R}} \gamma^{\mu} u_{R}\right)$ & $\mathcal{O}_{\phi u}^{(1)}$ & $\left(\phi^{\dagger} i D_{\mu} \phi\right)\left(\overline{d_{R}} \gamma^{\mu} d_{R}\right)$ & $\mathcal{O}_{\phi d}^{(1)}$ \\
\hline & $\left(\phi^{T} i \sigma_{2} i D_{\mu} \phi\right)\left(\overline{u_{R}} \gamma^{\mu} d_{R}\right)$ & $\mathcal{O}_{\phi u d}$ & & \\
\hline \multirow{4}{*}{ 捠 } & $\left(\overline{l_{L}} \sigma^{\mu \nu} e_{R}\right) \phi B_{\mu \nu}$ & $\mathcal{O}_{e B}$ & $\left(\overline{l_{L}} \sigma^{\mu \nu} e_{R}\right) \sigma^{a} \phi W_{\mu \nu}^{a}$ & $\mathcal{O}_{e W}$ \\
\hline & $\left(\overline{q_{L}} \sigma^{\mu \nu} u_{R}\right) \tilde{\phi} B_{\mu \nu}$ & $\mathcal{O}_{u B}$ & $\left(\overline{q_{L}} \sigma^{\mu \nu} u_{R}\right) \sigma^{a} \tilde{\phi} W_{\mu \nu}^{a}$ & $\mathcal{O}_{u W}$ \\
\hline & $\left(\overline{q_{L}} \sigma^{\mu \nu} d_{R}\right) \phi B_{\mu \nu}$ & $\mathcal{O}_{d B}$ & $\left(\overline{q_{L}} \sigma^{\mu \nu} d_{R}\right) \sigma^{a} \phi W_{\mu \nu}^{a}$ & $\mathcal{O}_{d W}$ \\
\hline & $\left(\overline{q_{L}} \sigma^{\mu \nu} T_{A} u_{R}\right) \tilde{\phi} G_{\mu \nu}^{A}$ & $\mathcal{O}_{u G}$ & $\left(\overline{q_{L}} \sigma^{\mu \nu} T_{A} d_{R}\right) \phi G_{\mu \nu}^{A}$ & $\mathcal{O}_{d G}$ \\
\hline \multirow{2}{*}{ 岳 } & $\left(\phi^{\dagger} \phi\right)\left(\overline{l_{L}} \phi e_{R}\right)$ & $\mathcal{O}_{e \phi}$ & & \\
\hline & $\left(\phi^{\dagger} \phi\right)\left(\overline{q_{L}} \tilde{\phi} u_{R}\right)$ & $\mathcal{O}_{u \phi}$ & $\left(\phi^{\dagger} \phi\right)\left(\overline{q_{L}} \phi d_{R}\right)$ & $\mathcal{O}_{d \phi}$ \\
\hline \multirow{5}{*}{ 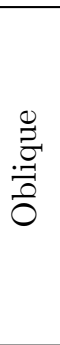 } & $\left(\phi^{\dagger} D_{\mu} \phi\right)\left(\left(D^{\mu} \phi\right)^{\dagger} \phi\right)$ & $\mathcal{O}_{\phi D}$ & & \\
\hline & $\phi^{\dagger} \phi B_{\mu \nu} B^{\mu \nu}$ & $\mathcal{O}_{\phi B}$ & $\phi^{\dagger} \phi \widetilde{B}_{\mu \nu} B^{\mu \nu}$ & $\mathcal{O}_{\phi \widetilde{B}}$ \\
\hline & $\phi^{\dagger} \phi W_{\mu \nu}^{a} W^{a \mu \nu}$ & $\mathcal{O}_{\phi W}$ & $\phi^{\dagger} \phi \widetilde{W}_{\mu \nu}^{a} W^{a \mu \nu}$ & $\mathcal{O}_{\phi \widetilde{W}}$ \\
\hline & $\phi^{\dagger} \sigma_{a} \phi W_{\mu \nu}^{a} B^{\mu \nu}$ & $\mathcal{O}_{W B}$ & $\phi^{\dagger} \sigma_{a} \phi \widetilde{W}_{\mu \nu}^{a} B^{\mu \nu}$ & $\mathcal{O}_{\widetilde{W} B}$ \\
\hline & $\phi^{\dagger} \phi G_{\mu \nu}^{A} G^{A \mu \nu}$ & $\mathcal{O}_{\phi G}$ & $\phi^{\dagger} \phi \widetilde{G}_{\mu \nu}^{A} G^{A \mu \nu}$ & $\mathcal{O}_{\phi \widetilde{G}}$ \\
\hline \multirow{2}{*}{ 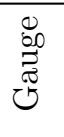 } & $\varepsilon_{a b c} W_{\mu}^{a \nu} W_{\nu}^{b \rho} W_{\rho}^{c \mu}$ & $\mathcal{O}_{W}$ & $\varepsilon_{a b c} \widetilde{W}_{\mu}^{a \nu} W_{\nu}^{b \rho} W_{\rho}^{c \mu}$ & $\mathcal{O}_{\widetilde{W}}$ \\
\hline & $f_{A B C} G_{\mu}^{A \nu} G_{\nu}^{B \rho} G_{\rho}^{C \mu}$ & $\mathcal{O}_{G}$ & $f_{A B C} \widetilde{G}_{\mu}^{A \nu} G_{\nu}^{B \rho} G_{\rho}^{C \mu}$ & $\mathcal{O}_{\widetilde{G}}$ \\
\hline
\end{tabular}

Table 8. Basis of dimension-six operators used in our analysis: operators other than four-fermion contact interactions. Flavor indices are omitted. Operators in grey color do not arise in the integration of heavy scalars at the tree level.

a perturbative field redefinition:

$$
\begin{aligned}
\mathcal{Q}_{\phi}^{(1)}=\phi^{\dagger} \phi\left(D_{\mu} \phi\right)^{\dagger} D^{\mu} \phi= & \frac{1}{2} \mathcal{O}_{\phi \square}-\mu_{\phi}^{2} \mathcal{O}_{\phi 4}+3 \lambda_{\phi} \mathcal{O}_{\phi}+ \\
& +\frac{1}{2}\left(y_{i i}^{e}\left(\mathcal{O}_{e \phi}\right)_{i i}+y_{i i}^{d}\left(\mathcal{O}_{d \phi}\right)_{i i}+V_{i j}^{\dagger} y_{j j}^{u}\left(\mathcal{O}_{u \phi}\right)_{i j}+\text { h.c. }\right) .
\end{aligned}
$$

\section{B Operator coefficients in the effective Lagrangian}

In tables 9-27 we present, for each new type of scalar, the contributions to the coefficients of the different dimension-six operators that result upon integration of one scalar multiplet at the tree level. Those contributions that arise only in the case where the theory contains several scalars at the same time (in the same or different representations) are given in 
table 28. All these results are given in the basis in appendix A. In some cases, this requires performing algebraic manipulations and field redefinitions on the operators that result directly from the integration.

Tables 9-28 also contain the interactions in the high-energy Lagrangian, using the notation of eq. (2.5). We only write here those interactions that are relevant for the computation of $\mathcal{L}_{\mathrm{Eff}}^{(6)}$. When gauge indices are explicitly shown, we use the following labeling for $\mathrm{SU}(2)_{L}$ indices in the different representations: $\alpha, \beta=\frac{1}{2},-\frac{1}{2}$ for $\mathrm{SU}(2)_{L}$ doublets; $a, b, c=1,2,3$ for the components of $\mathrm{SU}(2)_{L}$ triplets in Cartesian coordinates; and $I, J, K=$ $\frac{3}{2}, \frac{1}{2},-\frac{1}{2},-\frac{3}{2}$ for the components of the $\mathrm{SU}(2)_{L}$ quadruplets. The matrices used to construct the different invariants are the following:

- In constructing the triplets from two doublets we use the Pauli matrices

$$
\sigma_{1}=\left(\begin{array}{cc}
0 & 1 \\
1 & 0
\end{array}\right) ; \quad \sigma_{2}=\left(\begin{array}{rr}
0 & -i \\
i & 0
\end{array}\right) ; \quad \sigma_{3}=\left(\begin{array}{rr}
1 & 0 \\
0 & -1
\end{array}\right)
$$

- The isospin-1 product of two triplets is obtained through:

$$
f_{a b c}=\frac{i}{\sqrt{2}} \varepsilon_{a b c} .
$$

- Quadruplets are obtained from the product of an isospin-1 field and a doublet by means of

$$
\begin{gathered}
C_{a \beta}^{3 / 2}=\frac{1}{\sqrt{2}}\left(\begin{array}{rr}
1 & 0 \\
-i & 0 \\
0 & 0
\end{array}\right) ; \quad C_{a \beta}^{1 / 2}=\frac{1}{\sqrt{6}}\left(\begin{array}{rr}
0 & 1 \\
0 & -i \\
-2 & 0
\end{array}\right) ; \\
C_{a \beta}^{-1 / 2}=-\frac{1}{\sqrt{6}}\left(\begin{array}{cc}
1 & 0 \\
i & 0 \\
0 & 2
\end{array}\right) ; \quad C_{a \beta}^{-3 / 2}=-\frac{1}{\sqrt{2}}\left(\begin{array}{cc}
0 & 1 \\
0 & i \\
0 & 0
\end{array}\right) .
\end{gathered}
$$

- The singlet product of two quadruplets is obtained through the $\mathrm{SU}(2)$ product

$$
\epsilon_{I J}=\frac{1}{2}\left(\begin{array}{cccc}
0 & 0 & 0 & 1 \\
0 & 0 & -1 & 0 \\
0 & 1 & 0 & 0 \\
-1 & 0 & 0 & 0
\end{array}\right) .
$$

Finally, for $\mathrm{SU}(3)_{c}$ indices, we use the following notation for the symmetric product of colored fields:

$$
\psi_{1}^{(A \mid} \ldots \psi_{2}^{\mid B)} \equiv \frac{1}{2}\left(\psi_{1}^{A} \ldots \psi_{2}^{B}+\psi_{1}^{B} \ldots \psi_{2}^{A}\right) .
$$




\begin{tabular}{c}
$\mathcal{S} \sim(\mathbf{1}, \mathbf{1})_{\mathbf{0}}$ \\
$V_{\mathcal{S}}=\kappa_{\mathcal{S}} \mathcal{S} \phi^{\dagger} \phi+\lambda_{\mathcal{S}} \mathcal{S}^{2} \phi^{\dagger} \phi+\kappa_{\mathcal{S}^{3}} \mathcal{S}^{3}$ \\
\hline Dimension-Four Operators \\
$\alpha_{\phi 4}=\frac{\kappa_{\mathcal{S}}^{2}}{2 M_{\mathcal{S}}^{2}}$ \\
Scalar Operators \\
$\frac{\alpha_{\phi}}{\Lambda^{2}}=3 \frac{\kappa_{\mathcal{S}}^{2}}{M_{\mathcal{S}}^{2}}\left(-\frac{\lambda_{\mathcal{S}}}{M_{\mathcal{S}}^{2}}+\frac{\kappa_{\mathcal{S}} \kappa_{\mathcal{S}}}{M_{\mathcal{S}}^{4}}\right) \quad \frac{\alpha_{\phi \square}}{\Lambda^{2}}=-\frac{\kappa_{\mathcal{S}}^{2}}{2 M_{\mathcal{S}}^{4}}$ \\
\hline
\end{tabular}

Table 9. Operator coefficients arising from the integration of a $\mathcal{S}$ scalar field. See table 28 for collective contributions of several multiplets.

$$
\begin{gathered}
\mathcal{S}_{\mathbf{1}} \sim(\mathbf{1}, \mathbf{1})_{\mathbf{1}} \\
J_{\mathcal{S}_{1}}=\left(y_{\mathcal{S}_{1}}^{l}\right)_{i j} \overline{l_{L}^{i}} i \sigma_{2} l_{L}^{c j} \quad\left(\left(y_{\mathcal{S}_{1}}^{l}\right)_{i j}=-\left(y_{\mathcal{S}_{1}}^{l}\right)_{j i}\right) \\
\hline \text { Four-Fermion Operators: LLLL } \\
\frac{\left(\alpha_{l l}^{(1)}\right)_{i j k l}}{\Lambda^{2}}=2 \frac{\left(y_{\mathcal{S}_{1}}^{l}\right)_{i k}\left(y_{\mathcal{S}_{1}}^{l \dagger}\right)_{l j}}{M_{\mathcal{S}_{1}}^{2}}
\end{gathered}
$$

Table 10. Operator coefficients arising from the integration of a $\mathcal{S}_{1}$ scalar field.

\begin{tabular}{c}
$\mathcal{S}_{\mathbf{2}} \sim(\mathbf{1}, \mathbf{1})_{\mathbf{2}}$ \\
$J_{\mathcal{S}_{2}}=\left(y_{\mathcal{S}_{2}}^{e}\right)_{i j} \overline{e_{R}^{i}} e_{R}^{c j} \quad\left(\left(y_{\mathcal{S}_{2}}^{e}\right)_{i j}=\left(y_{\mathcal{S}_{2}}^{e}\right)_{j i}\right)$ \\
\hline Four-Fermion Operators: RRRR \\
$\frac{\left(\alpha_{e e}\right)_{i j k l}}{\Lambda^{2}}=\frac{\left(y_{\mathcal{S}_{2}}^{e}\right)_{k i}\left(y_{\mathcal{S}_{2}}^{e}\right)_{j l}}{M_{\mathcal{S}_{2}}^{2}}$ \\
\hline
\end{tabular}

Table 11. Operator coefficients arising from the integration of a $\mathcal{S}_{2}$ scalar field. 


\begin{tabular}{|c|c|}
\hline \multicolumn{2}{|c|}{$\begin{array}{l}\quad \boldsymbol{\varphi} \sim(\mathbf{1}, \mathbf{2})_{\frac{1}{2}} \\
\overline{e_{R}^{i}} l_{L}^{j}+\left(y_{\varphi}^{d}\right)_{i j} \overline{d_{R}^{i}} q_{L}^{j}+\left(y_{\varphi}^{u}\right)_{i j} i \sigma_{2}{\overline{q_{L}^{i}}}^{T} u_{R}^{j} \\
\left.\dagger^{\dagger} \phi\right)\left(\phi^{\dagger} \phi\right)+\text { h.c. }\end{array}$} \\
\hline $\begin{array}{l}\text { - LLRR } \\
\frac{\left(\alpha_{l e}\right)_{i j k l}}{\Lambda^{2}}=-\frac{\left(y_{\varphi}^{e}\right)_{k j}\left(y_{\varphi}^{e}\right)_{i l}}{2 M_{\varphi}^{2}} \\
\frac{\left(\alpha_{q d}^{(1)}\right)_{i j k l}}{\Lambda^{2}}=-\frac{\left(y_{\varphi}^{d}\right)_{k j}\left(y_{\varphi}^{d}\right)_{i l}}{6 M_{\varphi}^{2}} \\
\frac{\left(\alpha_{q u}^{(1)}\right)_{i j k l}}{\Lambda^{2}}=-\frac{\left(y_{\varphi}^{u}\right)_{i l}\left(y_{\varphi}^{u}{ }^{\dagger}\right)_{k j}}{6 M_{\varphi}^{2}} \\
\text { - LRRL } \\
\frac{\left(\alpha_{l e d q}\right)_{i j k l}}{\Lambda^{2}}=\frac{\left(y_{\varphi}^{d}\right)_{k l}\left(y_{\varphi}^{e}\right)_{i j}}{M_{\varphi}^{2}}\end{array}$ & $\begin{array}{l}\frac{\left(\alpha_{q d}^{(8)}\right)_{i j k l}}{\Lambda^{2}}=6 \frac{\left(\alpha_{q d}^{(1)}\right)_{i j k l}}{\Lambda^{2}} \\
\frac{\left(\alpha_{q u}^{(8)}\right)_{i j k l}}{\Lambda^{2}}=6 \frac{\left(\alpha_{q u}^{(1)}\right)_{i j k l}}{\Lambda^{2}} \\
\text { - LRLR } \\
\frac{\left(\alpha_{l e q u}\right)_{i j k l}}{\Lambda^{2}}=\frac{\left(y_{\varphi}^{u}\right)_{k l}\left(y_{\varphi}^{e}\right)_{i j}}{M_{\varphi}^{2}} \\
\frac{\left(\alpha_{q u d}^{(1)}\right)_{i j k l}}{\Lambda^{2}}=-\frac{\left(y_{\varphi}^{u}\right)_{i j}\left(y_{\varphi}^{d \dagger}\right)_{k l}}{M_{\varphi}^{2}}\end{array}$ \\
\hline $\begin{array}{c}\text { Scalar-1 } \\
\frac{\left(\alpha_{e \phi}\right)_{i j}}{\Lambda^{2}}=\frac{\lambda_{\varphi}\left(y_{\varphi}^{e}\right)_{i j}}{M_{\varphi}^{2}} \\
\frac{\left(\alpha_{u \phi}\right)_{i j}}{\Lambda^{2}}=-\frac{\lambda_{\varphi}^{*}\left(y_{\varphi}^{u}\right)_{i j}}{M_{\varphi}^{2}}\end{array}$ & $\frac{\left(\alpha_{d \phi}\right)_{i j}}{\Lambda^{2}}=\frac{\lambda_{\varphi}\left(y_{\varphi}^{d \dagger}\right)_{i j}}{M_{\varphi}^{2}}$ \\
\hline & \\
\hline
\end{tabular}

Table 12. Operator coefficients arising from the integration of a $\varphi$ scalar field. See table 28 for collective contributions of several multiplets.

\begin{tabular}{|c|c|c|}
\hline \multicolumn{3}{|c|}{ 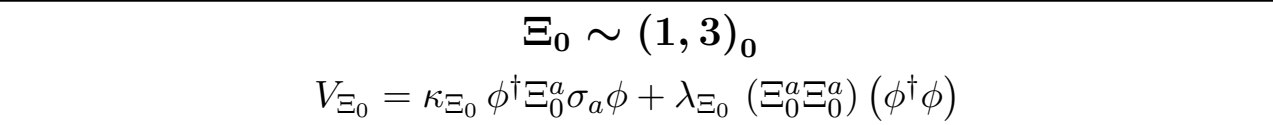 } \\
\hline \multicolumn{3}{|c|}{$\begin{array}{l}\text { Dimension-Four Operators } \\
\alpha_{\phi 4}=\frac{\kappa_{\Xi_{0}}^{2}}{2 M_{\Xi_{0}}^{2}}\left(1-4 \frac{\mu_{\phi}^{2}}{M_{\Xi_{0}}^{2}}\right)\end{array}$} \\
\hline$\frac{\left(\alpha_{e \phi}\right)_{i j}}{\Lambda^{2}}=\frac{\kappa_{\Xi_{0}}^{2} y_{i i}^{e}}{M_{\Xi_{0}}^{4}} \delta_{i j}$ & $\begin{array}{l}\text { lar-Fermion Ope } \\
\frac{\left(\alpha_{u \phi}\right)_{i j}}{\Lambda^{2}}=\frac{\kappa_{\Xi_{0}}^{2} V_{i j}^{\dagger} y_{j j}^{u}}{M_{\Xi_{0}}^{4}}\end{array}$ & 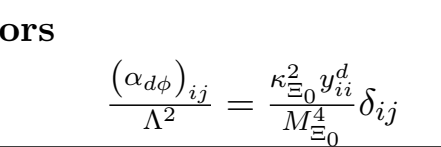 \\
\hline $\begin{array}{l}\text { Oblique Operators } \\
\frac{\alpha_{\phi D}}{\Lambda^{2}}=-2 \frac{\kappa_{\Xi_{0}}^{2}}{M_{\Xi_{0}}^{4}}\end{array}$ & & $\begin{array}{c}\text { Scalar Operators } \\
\frac{\alpha_{\phi \square}}{\Lambda^{2}}=\frac{\kappa_{\Xi_{0}}^{2}}{2 M_{\Xi_{0}}^{4}} \\
\frac{\alpha_{\phi}}{\Lambda^{2}}=-3 \frac{\kappa_{\Xi_{0}}^{2}}{M_{\Xi_{0}}^{4}}\left(\lambda_{\Xi_{0}}-2 \lambda_{\phi}\right)\end{array}$ \\
\hline
\end{tabular}

Table 13. Operator coefficients arising from the integration of a $\Xi_{0}$ scalar field. See table 28 for collective contributions of several multiplets. 


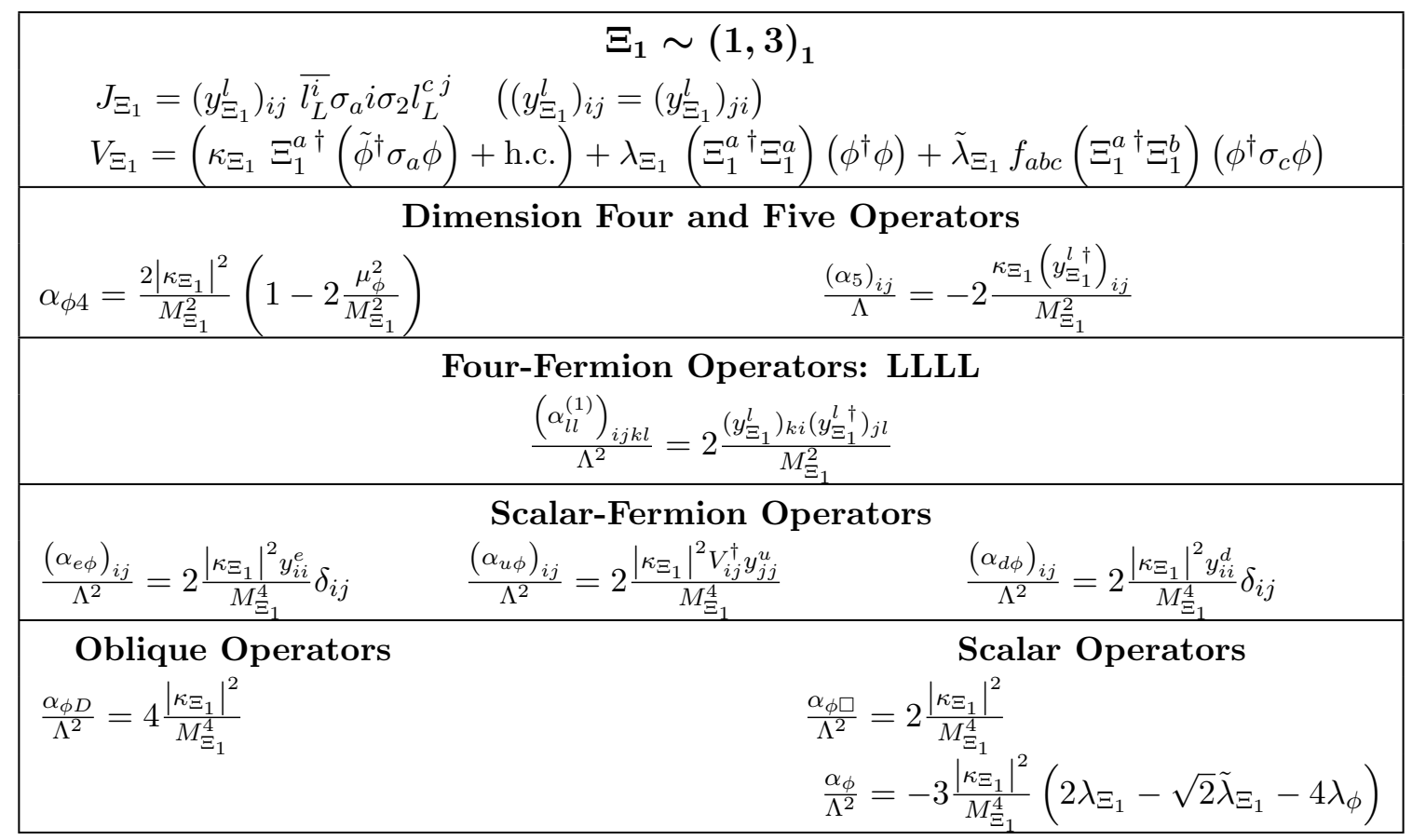

Table 14. Operator coefficients arising from the integration of a $\Xi_{1}$ scalar field. See table 28 for collective contributions of several multiplets.

$$
\begin{array}{|c|}
\hline \Theta_{1} \sim(1,4)_{\frac{1}{2}} \\
V_{\Theta_{1}}=\lambda_{\Theta_{1}}\left(\phi^{\dagger} \sigma_{a} \phi\right) C_{a \beta}^{I} \tilde{\phi}_{\beta} \epsilon_{I J} \Theta_{1}^{J}+\text { h.c. } \\
\hline \text { Scalar Operators } \\
\frac{\alpha_{\phi}}{\Lambda^{2}}=\frac{1}{2} \frac{\left|\lambda_{\Theta_{1}}\right|^{2}}{M_{\Theta_{1}}^{2}} \\
\hline
\end{array}
$$

Table 15. Operator coefficients arising from the integration of a $\Theta_{1}$ scalar field. See table 28 for collective contributions of several multiplets.

$$
\begin{gathered}
\Theta_{3} \sim(1,4)_{\frac{3}{2}} \\
V_{\Theta_{3}}=\lambda_{\Theta_{3}}\left(\phi^{\dagger} \sigma_{a} \tilde{\phi}\right) C_{a \beta}^{I} \tilde{\phi}_{\beta} \epsilon_{I J} \Theta_{3}^{J}+\text { h.c. } \\
\hline \text { Scalar Operators } \\
\frac{\alpha_{\phi}}{\Lambda^{2}}=\frac{3}{2} \frac{\left|\lambda_{\Theta_{3}}\right|^{2}}{M_{\Theta_{3}}^{2}} \\
\hline
\end{gathered}
$$

Table 16. Operator coefficients arising from the integration of a $\Theta_{3}$ scalar field. See table 28 for collective contributions of several multiplets. 


\begin{tabular}{|c|c|}
\hline \multicolumn{2}{|c|}{$\begin{aligned} \boldsymbol{\omega}_{\mathbf{1}} \sim(\mathbf{3}, \mathbf{1})_{-\frac{1}{3}} & \\
J_{\omega_{1}} & =\left(y_{\omega_{1}}^{q l}\right)_{i j} \overline{q_{L}^{c i}} i \sigma_{2} l_{L}^{j}+\left(y_{\omega_{1}}^{q q}\right)_{i j} \varepsilon_{A B C} \overline{q_{L}^{i B}} i \sigma_{2} q_{L}^{c j} C^{q q}+\left(y_{\omega_{1}}^{e u}\right)_{i j} \overline{e_{R}^{c i}} u_{R}^{j}+\left(y_{\omega_{1}}^{d u}\right)_{i j} \varepsilon_{A B C} \overline{d_{R}^{i B}} u_{R}^{c j} c \\
\left(\left(y_{\omega_{1}}^{q q}\right)_{i j}\right. & \left.=\left(y_{\omega_{1}}^{q q}\right)_{j i}\right)\end{aligned}$} \\
\hline & Operators: \\
\hline $\begin{array}{l}\text { - LLLL } \\
\frac{\left(\alpha_{l q}^{(1)}\right)_{i j k l}}{\Lambda^{2}}=\frac{1}{4} \frac{\left(y_{\omega_{1}}^{q l}\right)_{l j}\left(y_{\omega_{1}}^{q l \dagger}\right)_{i k}}{M_{\omega_{1}}^{2}} \\
\frac{\left(\alpha_{l q}^{(3)}\right)_{i j k l}}{\Lambda^{2}}=-\frac{\left(\alpha_{l q}^{(1)}\right)_{i j k l}}{\Lambda^{2}} \\
\frac{\left(\alpha_{q q}^{(1)}\right)_{i j k l}}{\Lambda^{2}}=\frac{4}{3} \frac{\left(y_{\omega_{1}}^{q q}\right)_{k i}\left(y_{\omega_{1}}^{q \dagger}\right)_{j l}}{M_{\omega_{1}}^{2}} \\
\frac{\left(\alpha_{q q}^{(8)}\right)_{i j k l}}{\Lambda^{2}}=-3 \frac{\left(\alpha_{q q}^{(1)}\right)_{i j k l}}{\Lambda^{2}}\end{array}$ & $\begin{array}{l}\text { - RRRR } \\
\frac{\left(\alpha_{u d}^{(1)}\right)_{i j k l}}{\Lambda^{2}}=\frac{1}{3} \frac{\left(y_{\omega_{1}}^{d u}\right)_{k i}\left(y_{\omega_{1}}^{d u}\right)_{j l}}{M_{\omega_{1}}^{2}} \\
\frac{\left(\alpha_{u d}^{(8)}\right)_{i j k l}}{\Lambda^{2}}=-3 \frac{\left(\alpha_{u d}^{(1)}\right)_{i j k l}}{\Lambda^{2}} \\
\frac{\left(\alpha_{e u}\right)_{i j k l}}{\Lambda^{2}}=\frac{1}{2} \frac{\left(y_{\omega_{1}}^{e u}\right)_{j l}\left(y_{\omega_{1}}^{e u}\right)_{k i}}{M_{\omega_{1}}^{2}}\end{array}$ \\
\hline - LRLR & - В \& \& L \\
\hline$\frac{\left(\alpha_{q u d}^{(1)}\right)_{i j k l}}{\Lambda^{2}}=\frac{4}{3} \frac{\left(y_{\omega_{1}}^{q q}\right)_{k i}\left(y_{\omega_{1}}^{d u}\right)_{j l}}{M_{\omega_{1}}^{2}}$ & $\frac{\left(\alpha_{l q d u}\right)_{i j k l}}{\Lambda^{2}}=-\frac{\left(y_{\omega_{1}}^{d u}\right)_{k l}\left(y_{\omega_{1}}^{q l \dagger}\right)_{i j}}{M_{\omega_{1}}^{2}}$ \\
\hline$\frac{\left(\alpha_{q u d}^{(8)}\right)_{i j k l}}{\Lambda^{2}}=-3 \frac{\left(\left(_{q u d}^{(1)}\right)_{i j k l}\right.}{\Lambda^{2}}$ & $\frac{\left(\alpha_{q q e u}\right)_{i j k l}}{\Lambda^{2}}=\frac{\left(y_{\omega_{1}}^{q q}\right)_{j i}\left(y_{\omega_{1}}^{e u \dagger}\right)_{l k}}{M_{\omega_{1}}^{2}}$ \\
\hline $\begin{aligned} \frac{\left(\alpha_{l u q e}\right)_{i j k l}}{\Lambda^{2}} & =\frac{\left(y_{\omega_{1}}^{e u}\right)_{l j}\left(y_{\omega_{1}}^{q l}\right)_{i k}}{M_{\omega_{1}}^{2}} \\
\frac{\left(\alpha_{l e q u}\right)_{i j k l}}{\Lambda^{2}} & =\frac{\left(\alpha_{l u q e}\right)_{i l k j}}{\Lambda^{2}}\end{aligned}$ & $\begin{aligned} \frac{\left(\alpha_{l q q q}^{(1)}\right)_{i j k l}}{\Lambda^{2}} & =-\frac{\left(y_{\omega_{1}}^{q l \dagger}\right)_{i j}\left(y_{\omega_{1}}^{q q}\right)_{k l}}{M_{\omega_{1}}^{2}} \\
\frac{\left(\alpha_{u d e u}\right)_{i j k l}}{\Lambda^{2}} & =-\frac{\left(y_{\omega_{1}}^{e u}\right)_{l k}\left(y_{\omega_{1}}^{d u}\right)_{j i}}{M_{\omega_{1}}^{2}}\end{aligned}$ \\
\hline
\end{tabular}

Table 17. Operator coefficients arising from the integration of a $\omega_{1}$ scalar field.

$$
\begin{gathered}
\boldsymbol{\omega}_{\mathbf{2}} \sim(\mathbf{3}, \mathbf{1})_{\frac{\mathbf{2}}{3}} \\
J_{\omega_{2}}=\left(y_{\omega_{2}}^{d}\right)_{i j} \varepsilon_{A B C} \overline{d_{R}^{i B}} d_{R}^{c j} C \quad\left(\left(y_{\omega_{2}}^{d}\right)_{i j}=-\left(y_{\omega_{2}}^{d}\right)_{j i}\right) \\
\hline \text { Four-Fermion Operators: RRRR } \\
\frac{\left(\alpha_{d d}^{(1)}\right)_{i j k l}}{\Lambda^{2}}=2 \frac{\left(y_{\omega_{2}}^{d}\right)_{k i}\left(y_{\omega_{2}}^{d \dagger}\right)_{j l}}{M_{\omega_{2}}^{2}} \\
\hline
\end{gathered}
$$

Table 18. Operator coefficients arising from the integration of a $\omega_{2}$ scalar field.

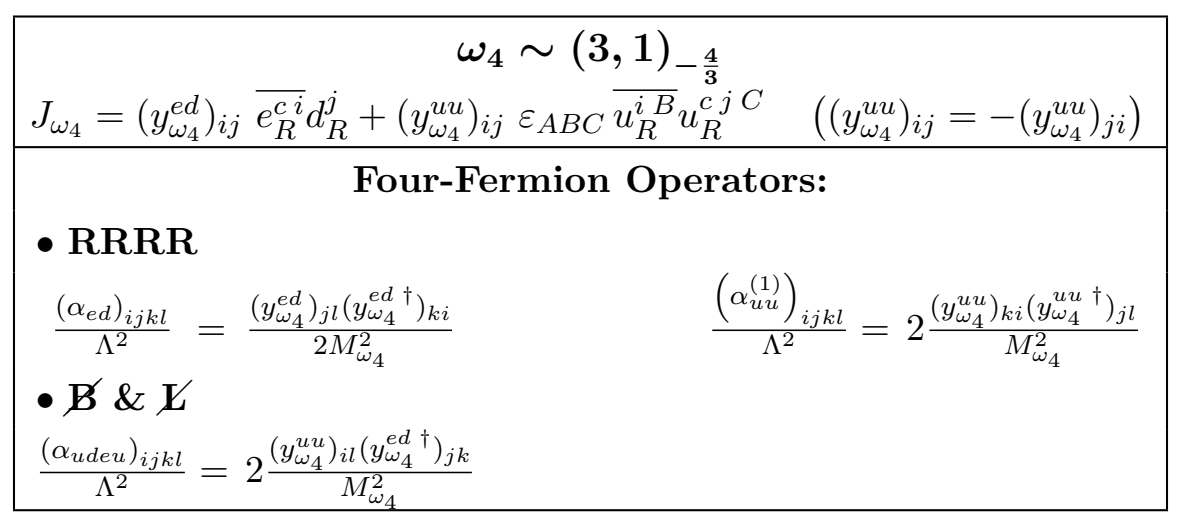

Table 19. Operator coefficients arising from the integration of a $\omega_{4}$ scalar field. 


\begin{tabular}{|c|}
\hline$\Pi_{\mathbf{1}} \sim(\mathbf{3}, \mathbf{2})_{\frac{1}{6}}$ \\
$J_{\Pi_{1}}=\left(y_{\Pi_{1}}^{l d}\right)_{i j} i \sigma_{2} \frac{l}{L}_{L}^{i} d_{R}^{j}$ \\
\hline $\begin{array}{c}\text { Four-Fermion Operators: LLRR } \\
\frac{\left(\alpha_{l d}\right)_{i j k l}}{\Lambda^{2}}=-\frac{\left(y_{\Pi_{1}}^{l d}\right)_{i l}\left(y_{\Pi_{1}}^{l d}\right)_{k j}}{2 M_{\Pi_{1}}^{2}}\end{array}$ \\
\hline
\end{tabular}

Table 20. Operator coefficients arising from the integration of a $\Pi_{1}$ scalar field.

\begin{tabular}{|c|c|}
\hline \multicolumn{2}{|c|}{$\begin{array}{l}\boldsymbol{\Pi}_{\mathbf{7}} \sim(\mathbf{3}, \mathbf{2})_{\frac{\mathbf{7}}{\mathbf{6}}} \\
)_{i j} i \sigma_{2}{\overline{l_{L}^{i}}}^{T} u_{R}^{j}+\left(y_{\Pi_{7}}^{e q}\right)_{i j} \overline{e_{R}^{i}} q_{L}^{j}\end{array}$} \\
\hline \multicolumn{2}{|c|}{ Four-Fermion Operators: } \\
\hline $\begin{array}{l}\text { - LLRR } \\
\frac{\left(\alpha_{l u}\right)_{i j k l}}{\Lambda^{2}}=-\frac{\left(y_{\Pi_{7}}^{l u}\right)_{i l}\left(y_{\Pi_{7}}^{l u \dagger}\right)_{k j}}{2 M_{\Pi_{7}}^{2}} \\
\frac{\left(\alpha_{q e}\right)_{i j k l}}{\Lambda^{2}}=-\frac{\left(y_{\Pi_{7}}^{e q}\right)_{k j}\left(y_{\Pi_{7}}^{e q}\right)_{i l}}{2 M_{\Pi_{7}}^{2}}\end{array}$ & $\begin{array}{l}\text { - LRLR } \\
\frac{\left(\alpha_{l u q e}\right)_{i j k l}}{\Lambda^{2}}=-\frac{\left(y_{\Pi_{7}}^{l u}\right)_{i j}\left(y_{\Pi_{7}}^{e q}\right)_{k l}}{M_{\Pi_{7}}^{2}}\end{array}$ \\
\hline
\end{tabular}

Table 21. Operator coefficients arising from the integration of a $\Pi_{7}$ scalar field.

\begin{tabular}{|c|c|}
\hline \multicolumn{2}{|c|}{ 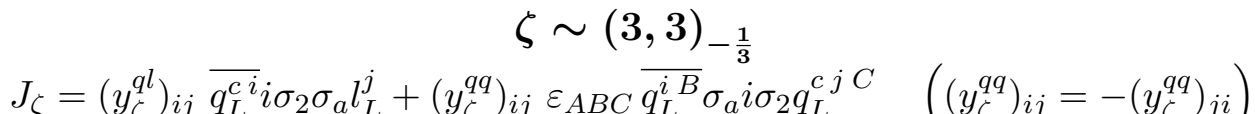 } \\
\hline $\begin{array}{l}\text { - LLLL } \\
\frac{\left(\alpha_{l q}^{(1)}\right)_{i j k l}}{\Lambda^{2}}=\frac{3}{4} \frac{\left(y_{\zeta}^{q l}\right)_{l j}\left(y_{\zeta}^{q l \dagger}\right)_{i k}}{M_{\zeta}^{2}} \\
\frac{\left(\alpha_{q q}^{(1)}\right)_{i j k l}}{\Lambda^{2}}=\frac{4}{3} \frac{\left(y_{\zeta}^{q q}\right)_{k i}\left(y_{\zeta}^{q q \dagger}\right)_{j l}}{M_{\zeta}^{2}} \\
\text { - B \& \& } \mathbf{L} \\
\frac{\left(\alpha_{l q q q}^{(3)}\right)_{i j k l}}{\Lambda^{2}}=-\frac{\left(y_{\zeta}^{q q}\right)_{k l}\left(y_{\zeta}^{q l \dagger}\right)_{i j}}{M_{\zeta}^{2}}\end{array}$ & $\begin{array}{l}\text { Operators: } \\
\begin{array}{l}\frac{\left(\alpha_{l q}^{(3)}\right)_{i j k l}}{\Lambda^{2}}=\frac{1}{3} \frac{\left(\alpha_{l q}^{(1)}\right)_{i j k l}}{\Lambda^{2}} \\
\frac{\left(\alpha_{q q}^{(8)}\right)_{i j k l}}{\Lambda^{2}}=-3 \frac{\left(\alpha_{q q}^{(1)}\right)_{i j k l}}{\Lambda^{2}}\end{array}\end{array}$ \\
\hline
\end{tabular}

Table 22. Operator coefficients arising from the integration of a $\zeta$ scalar field. 


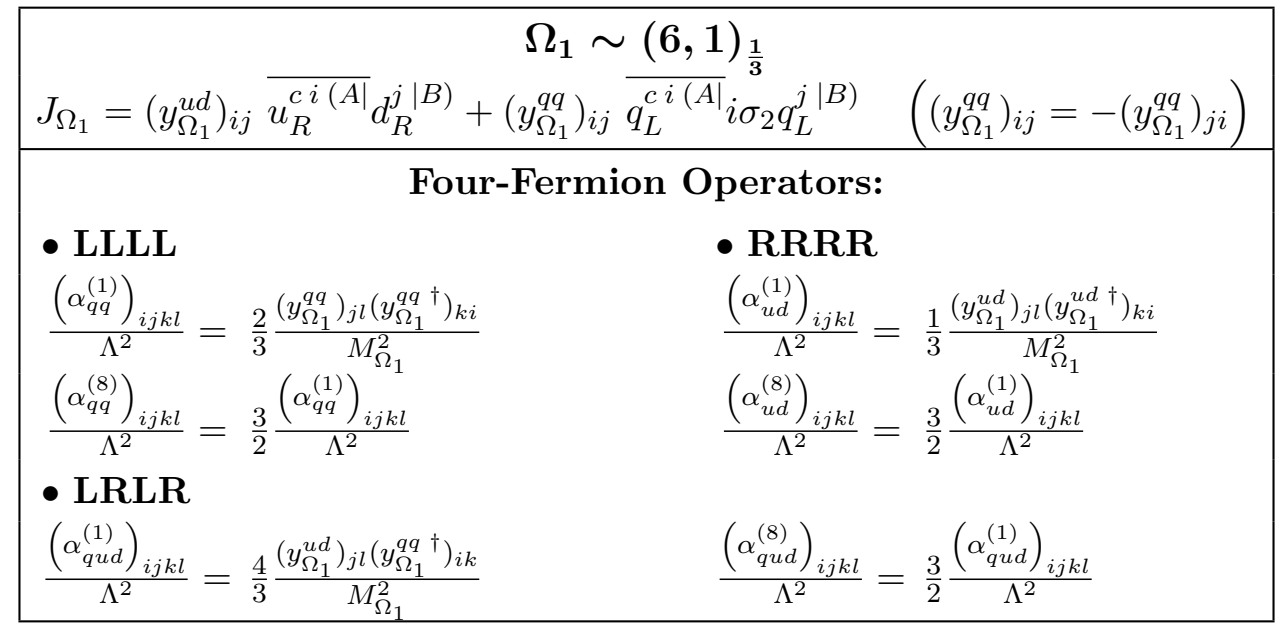

Table 23. Operator coefficients arising from the integration of a $\Omega_{1}$ scalar field.

$$
\begin{gathered}
\mathbf{\Omega}_{\mathbf{2}} \sim(\mathbf{6}, \mathbf{1})_{-\frac{2}{3}} \\
J_{\Omega_{2}}=\left(y_{\Omega_{2}}^{d}\right)_{i j} \frac{\left.d_{R}^{c i(A \mid} d_{R}^{j} \mid B\right)}{d_{R}}\left(\left(y_{\Omega_{2}}^{d}\right)_{i j}=\left(y_{\Omega_{2}}^{d}\right)_{j i}\right) \\
\hline \text { Four-Fermion Operators: RRRR } \\
\frac{\left(\alpha_{d d}^{(1)}\right)_{i j k l}}{\Lambda^{2}}=\frac{\left(y_{\Omega_{2}}^{d}\right)_{j l}\left(y_{\Omega_{2}}^{d \dagger}\right)_{k i}}{M_{\Omega_{2}}^{2}} \\
\hline
\end{gathered}
$$

Table 24. Operator coefficients arising from the integration of a $\Omega_{2}$ scalar field.

$$
\begin{gathered}
\mathbf{\Omega}_{\mathbf{4}} \sim(\mathbf{6 , 1})_{\frac{4}{3}} \\
J_{\Omega_{4}}=\left(y_{\Omega_{4}}^{u}\right)_{i j} \frac{u_{R}^{c i(A \mid}}{u_{R}^{j \mid B)}}\left(\left(y_{\Omega_{4}}^{u}\right)_{i j}=\left(y_{\Omega_{4}}^{u}\right)_{j i}\right) \\
\hline \text { Four-Fermion Operators: RRRR } \\
\frac{\left(\alpha_{u u}^{(1)}\right)_{i j k l}}{\Lambda^{2}}=\frac{\left(y_{\Omega_{4}}^{u}\right)_{j l}\left(y_{\Omega_{4}}^{u \dagger}\right)_{k i}}{M_{\Omega_{4}}^{2}} \\
\hline
\end{gathered}
$$

Table 25. Operator coefficients arising from the integration of a $\Omega_{4}$ scalar field. 


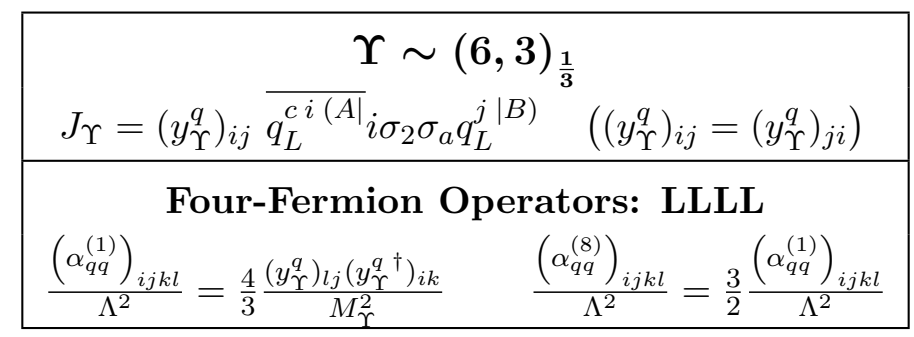

Table 26. Operator coefficients arising from the integration of a $\Upsilon$ scalar field.

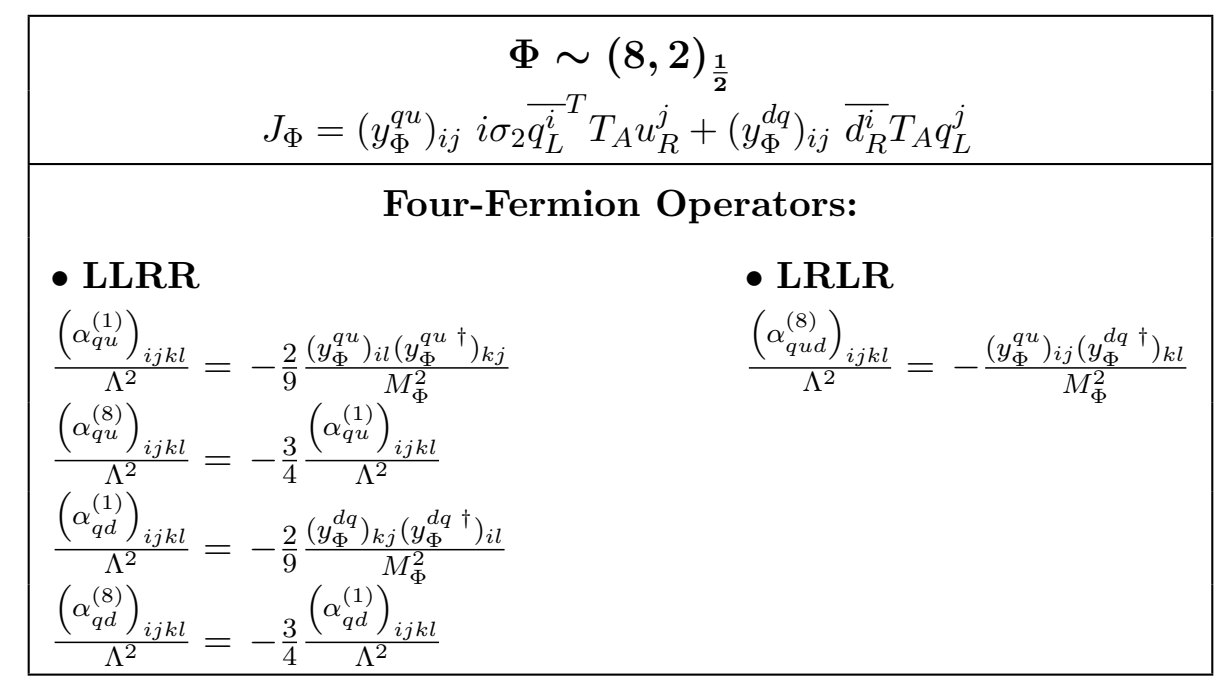

Table 27. Operator coefficients arising from the integration of a $\Phi$ scalar field. 


\begin{tabular}{|c|}
\hline $\begin{array}{l}\text { Mixed contributions from }\left\{\mathcal{S}, \varphi, \Xi_{0}, \Xi_{1}, \Theta_{1}, \Theta_{3}\right\} \\
\left.+\Xi_{1 i}^{a \dagger} J_{\Xi_{1 i}}^{a}+\text { h.c. }\right)-\kappa_{\mathcal{S}}^{i} \mathcal{S}_{i} \phi^{\dagger} \phi-\kappa_{\mathcal{S}^{3}}^{i j k} \mathcal{S}_{i} \mathcal{S}_{j} \mathcal{S}_{k}-\kappa_{\Xi_{0}}^{i} \Xi_{0 i}^{a} \phi^{\dagger} \sigma_{a} \phi \\
\left.i^{\dagger} \tilde{\phi}^{\dagger} \sigma_{a} \phi+\kappa_{\mathcal{S} \varphi}^{i j} \mathcal{S}_{i} \varphi_{j}^{\dagger} \phi+\text { h.c. }\right)-\kappa_{\mathcal{S} \Xi_{0}}^{i j k} \mathcal{S}_{i} \Xi_{0 j}^{a} \Xi_{0 k}^{a}-\kappa_{\mathcal{S} \Xi_{1}}^{i j k} \mathcal{S}_{i} \Xi_{1 j}^{a \dagger} \Xi_{1 k}^{a} \\
\Xi_{0 i}^{a} \Xi_{0 j}^{b} \Xi_{0 k}^{c}-\kappa_{\Xi_{0}}^{i j k} \Xi_{1} f_{a b c} \Xi_{0 i}^{a} \Xi_{1 j}^{b \dagger} \Xi_{1 k}^{c}-\left(\kappa_{\Xi_{0} \varphi}^{i j} \Xi_{0 i}^{a}\left(\varphi_{j}^{\dagger} \sigma_{a} \phi\right)+\kappa_{\Xi_{1} \varphi}^{i j} \Xi_{1 i}^{a \dagger}\left(\tilde{\varphi}_{j}^{\dagger} \sigma_{a} \phi\right)+\text { h.c. }\right) \\
\left.\Xi_{0 i}^{a} C_{a \beta}^{I} \tilde{\phi}_{\beta} \epsilon_{I J} \Theta_{1 j}^{J}+\kappa_{\Xi_{1}}^{i j} \Theta_{1} \Xi_{1 i}^{a \dagger} C_{a \beta}^{I} \phi_{\beta} \epsilon_{I J} \Theta_{1 j}^{J}+\kappa_{\Xi_{1}}^{i j} \Theta_{3} \Xi_{1 i}^{a \dagger} C_{a \beta}^{I} \tilde{\phi}_{\beta} \epsilon_{I J} \Theta_{3 j}^{J}+\text { h.c. }\right) \\
\left(\phi^{\dagger} \phi\right)-\left(\lambda_{\varphi}^{i}\left(\varphi_{i}^{\dagger} \phi\right)\left(\phi^{\dagger} \phi\right)+\text { h.c. }\right)-\lambda_{\Xi_{0}}^{i j} \Xi_{0 i}^{a} \Xi_{0 j}^{a}\left(\phi^{\dagger} \phi\right)-\tilde{\lambda}_{\Xi_{0}}^{i j} \Xi_{0 i}^{a} \Xi_{0 j}^{b} f_{a b c}\left(\phi^{\dagger} \sigma_{c} \phi\right) \\
\Xi_{1 j}^{a}\left(\phi^{\dagger} \phi\right)-\tilde{\lambda}_{\Xi_{1}}^{i j} f_{a b c} \Xi_{1 i}^{a \dagger} \Xi_{1 j}^{b}\left(\phi^{\dagger} \sigma_{c} \phi\right)-\lambda_{\mathcal{S} \Xi_{0}}^{i j} \mathcal{S}_{i} \Xi_{0 j}^{a}\left(\phi^{\dagger} \sigma_{a} \phi\right) \\
\left.{ }_{i} \Xi_{1 j}^{a \dagger}\left(\tilde{\phi}^{\dagger} \sigma_{a} \phi\right)+\lambda_{\Xi_{1} \Xi_{0}}^{i j} f_{a b c} \Xi_{1 i}^{a \dagger} \Xi_{0 j}^{b}\left(\tilde{\phi}^{\dagger} \sigma_{c} \phi\right)+\text { h.c. }\right) \\
\left.\left.\phi^{\dagger} \sigma_{a} \phi\right) C_{a \beta}^{I} \tilde{\phi}_{\beta} \epsilon_{I J} \Theta_{1 i}^{J}+\lambda_{\Theta_{3}}^{i}\left(\phi^{\dagger} \sigma_{a} \tilde{\phi}\right) C_{a \beta}^{I} \tilde{\phi}_{\beta} \epsilon_{I J} \Theta_{3 i}^{J}+\text { h.c. }\right)\end{array}$ \\
\hline 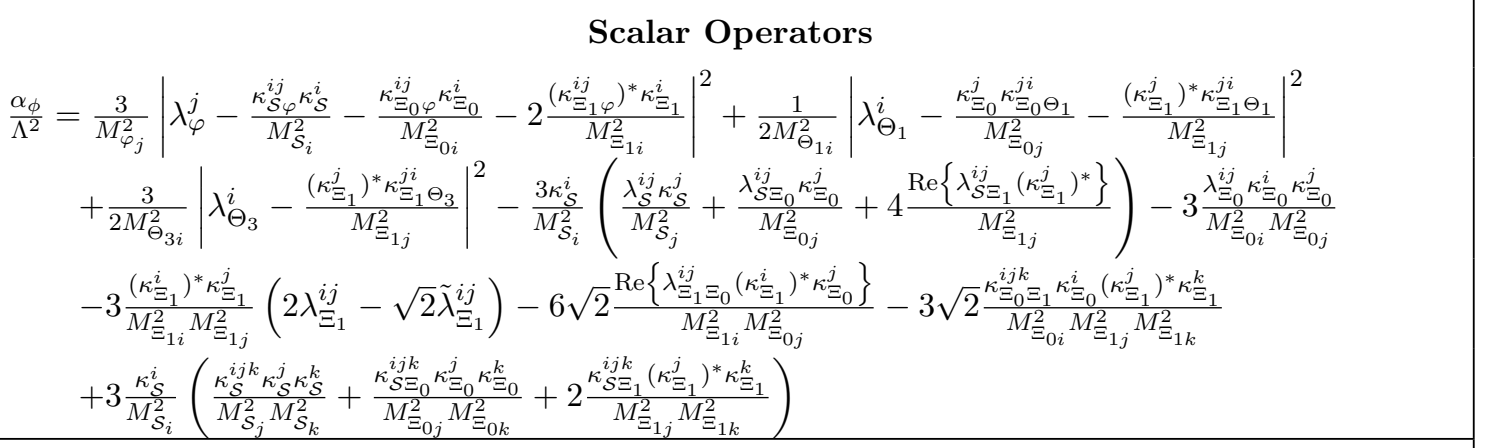 \\
\hline $\begin{array}{c}\text { Scalar-Fermion Operators } \\
\frac{\left(\alpha_{e \phi}\right)_{i j}}{\Lambda^{2}}=\frac{1}{M_{\varphi_{j}}^{2}}\left(\lambda_{\varphi}^{j}-\frac{\kappa_{\mathcal{S}}^{i} \kappa_{\mathcal{S} \varphi}^{i j}}{M_{\mathcal{S}_{i}}^{2}}-\frac{\kappa_{\Xi_{0}}^{i} \kappa_{\Xi_{0}}^{i j}}{M_{\Xi_{0 i}}^{2}}-2 \frac{\kappa_{\Xi_{1}}^{i}\left(\kappa_{\Xi_{1 \varphi}}^{i j}\right)^{*}}{M_{\Xi_{1 i}}^{2}}\right)\left(y_{\varphi}^{e}\right)_{i j} \\
\frac{\left(\alpha_{d \phi}\right)_{i j}}{\Lambda^{2}}=\frac{1}{M_{\varphi_{j}}^{2}}\left(\lambda_{\varphi}^{j}-\frac{\kappa_{\mathcal{S}}^{i} \kappa_{\mathcal{S}}^{i j}}{M_{\mathcal{S}_{i}}^{2}}-\frac{\kappa_{\Xi_{0}}^{i} \kappa_{\Xi_{0}}^{i j}}{M_{\Xi_{0 i}}^{2}}-2 \frac{\kappa_{\Xi_{\Xi_{1}}}^{i}\left(\kappa_{\Xi_{1 \varphi}}^{i j}\right)^{*}}{M_{\Xi_{1}}^{2}}\right)\left(y_{\varphi}^{d \dagger}\right)_{i j} \\
\frac{\left(\alpha_{u \phi}\right)_{i j}}{\Lambda^{2}}=-\frac{1}{M_{\varphi_{j}}^{2}}\left(\left(\lambda_{\varphi}^{j}\right)^{*}-\frac{\kappa_{\mathcal{S}}^{i}\left(\kappa_{\mathcal{S}}^{i j}\right)^{*}}{M_{\mathcal{S}_{i}}^{i j}}-\frac{\kappa_{\Xi_{0}}^{i}\left(\kappa_{\Xi_{0}}^{i j}\right)^{*}}{M_{\Xi_{0 i}}^{*}}-2 \frac{\left(\kappa_{\Xi_{1}}^{i}\right)^{*} \kappa_{\Xi_{1 \varphi}}^{i j}}{M_{\Xi_{1 i}}^{2}}\right)\left(y_{\varphi}^{u}\right)_{i j}\end{array}$ \\
\hline
\end{tabular}

Table 28. Collective contributions from several scalars to the dimension six effective Lagrangian. Only those operators that receive contributions from interactions involving more than one scalar at a time are shown. The full contribution to the operator coefficient is presented in that case. For the other operators the contributions can be read by adding the corresponding pieces from the tables obtained integrating one scalar at a time. Note that, due to the antisymmetric properties of the interaction, the couplings $\kappa_{\Xi_{0}^{3}}^{i j k}$ and $\tilde{\lambda}_{\Xi_{0}}^{i j}$ do not contribute to $\mathcal{L}_{6}$. 
Open Access. This article is distributed under the terms of the Creative Commons Attribution License (CC-BY 4.0), which permits any use, distribution and reproduction in any medium, provided the original author(s) and source are credited.

\section{References}

[1] ATLAS collaboration, Observation of a new particle in the search for the Standard Model Higgs boson with the ATLAS detector at the LHC, Phys. Lett. B 716 (2012) 1 [arXiv: 1207.7214] [INSPIRE].

[2] CMS collaboration, Observation of a new boson at a mass of $125 \mathrm{GeV}$ with the CMS experiment at the LHC, Phys. Lett. B 716 (2012) 30 [arXiv:1207.7235] [INSPIRE].

[3] F. del Aguila, M. Pérez-Victoria and J. Santiago, Effective description of quark mixing, Phys. Lett. B 492 (2000) 98 [hep-ph/0007160] [INSPIRE].

[4] A. Pomarol and F. Riva, Towards the Ultimate SM Fit to Close in on Higgs Physics, JHEP 01 (2014) 151 [arXiv: 1308.2803] [INSPIRE].

[5] F. del Aguila, M. Pérez-Victoria and J. Santiago, Observable contributions of new exotic quarks to quark mixing, JHEP 09 (2000) 011 [hep-ph/0007316] [INSPIRE].

[6] F. del Aguila, J. de Blas and M. Pérez-Victoria, Effects of new leptons in Electroweak Precision Data, Phys. Rev. D 78 (2008) 013010 [arXiv:0803.4008] [INSPIRE].

[7] F. del Aguila, J. de Blas and M. Pérez-Victoria, Electroweak Limits on General New Vector Bosons, JHEP 09 (2010) 033 [arXiv:1005.3998] [INSPIRE].

[8] V. Silveira and A. Zee, Scalar Phantoms, Phys. Lett. B 161 (1985) 136 [InSPIRE].

[9] J.F. Nieves, Baryon and Lepton Number Nonconserving Processes and Intermediate Mass Scales, Nucl. Phys. B 189 (1981) 182 [inSPIRE].

[10] W. Buchmüller, R. Ruckl and D. Wyler, Leptoquarks in Lepton-Quark Collisions, Phys. Lett. B 191 (1987) 442 [Erratum ibid. B 448 (1999) 320] [INSPIRE].

[11] E. Ma, M. Raidal and U. Sarkar, Probing the exotic particle content beyond the standard model, Eur. Phys. J. C 8 (1999) 301 [hep-ph/9808484] [INSPIRE].

[12] E. Del Nobile, R. Franceschini, D. Pappadopulo and A. Strumia, Minimal Matter at the Large Hadron Collider, Nucl. Phys. B 826 (2010) 217 [arXiv:0908.1567] [INSPIRE].

[13] T. Han, I. Lewis and Z. Liu, Colored Resonant Signals at the LHC: Largest Rate and Simplest Topology, JHEP 12 (2010) 085 [arXiv: 1010.4309] [INSPIRE].

[14] J.A. Aguilar-Saavedra and M. Pérez-Victoria, Probing the Tevatron t $\bar{t}$ asymmetry at LHC, JHEP 05 (2011) 034 [arXiv: 1103.2765] [InSPIRE].

[15] B. Grinstein, A.L. Kagan, J. Zupan and M. Trott, Flavor Symmetric Sectors and Collider Physics, JHEP 10 (2011) 072 [arXiv:1108.4027] [INSPIRE].

[16] W. Skiba, TASI Lectures on Effective Field Theory and Precision Electroweak Measurements, arXiv: 1006.2142 [INSPIRE].

[17] Z.U. Khandker, D. Li and W. Skiba, Electroweak Corrections from Triplet Scalars, Phys. Rev. D 86 (2012) 015006 [arXiv:1201.4383] [INSPIRE].

[18] B. Henning, X. Lu and H. Murayama, How to use the Standard Model effective field theory, arXiv: 1412.1837 [INSPIRE]. 
[19] I. Dorsner, S. Fajfer and N. Kosnik, Heavy and light scalar leptoquarks in proton decay, Phys. Rev. D 86 (2012) 015013 [arXiv: 1204.0674] [INSPIRE].

[20] F. del Aguila, M. Chala, J. Santiago and Y. Yamamoto, Collider limits on leptophilic interactions, arXiv:1411.7394 [INSPIRE].

[21] S. Weinberg, Baryon and Lepton Nonconserving Processes, Phys. Rev. Lett. 43 (1979) 1566 [INSPIRE].

[22] F. del Aguila, J.A. Aguilar-Saavedra, J. de Blas and M. Zralek, Looking for signals beyond the neutrino Standard Model, Acta Phys. Polon. B 38 (2007) 3339 [arXiv:0710.2923] [INSPIRE].

[23] F. del Aguila, J.A. Aguilar-Saavedra, J. de Blas and M. Pérez-Victoria, Electroweak constraints on see-saw messengers and their implications for LHC, arXiv:0806.1023 [INSPIRE].

[24] F. del Aguila, J.A. Aguilar-Saavedra and J. de Blas, New neutrino interactions at large colliders, PoS(ICHEP 2010) 296 [arXiv: 1012.1327] [INSPIRE].

[25] M.E. Peskin and T. Takeuchi, Estimation of oblique electroweak corrections, Phys. Rev. D 46 (1992) 381 [INSPIRE].

[26] J. de Blas et al., Global Bayesian Analysis of the Higgs-boson Couplings, arXiv:1410.4204 [INSPIRE].

[27] J.R. Ellis, V. Sanz and T. You, The Effective Standard Model after LHC Run I, arXiv: 1410.7703 [INSPIRE].

[28] J. Baglio, A. Djouadi, R. Gröber, M.M. Mühlleitner, J. Quevillon and M. Spira, The measurement of the Higgs self-coupling at the LHC: theoretical status, JHEP 04 (2013) 151 [arXiv: 1212.5581] [INSPIRE].

[29] O. Domenech, A. Pomarol and J. Serra, Probing the SM with Dijets at the LHC, Phys. Rev. D 85 (2012) 074030 [arXiv:1201.6510] [InSPIRE].

[30] G. Isidori, Y. Nir and G. Perez, Flavor Physics Constraints for Physics Beyond the Standard Model, Ann. Rev. Nucl. Part. Sci. 60 (2010) 355 [arXiv:1002.0900] [INSPIRE].

[31] M. Carpentier and S. Davidson, Constraints on two-lepton, two quark operators, Eur. Phys. J. C 70 (2010) 1071 [arXiv: 1008.0280] [INSPIRE].

[32] J. de Blas, M. Chala and J. Santiago, Global Constraints on Lepton-Quark Contact Interactions, Phys. Rev. D 88 (2013) 095011 [arXiv:1307.5068] [InSPIRE].

[33] J.A. Aguilar-Saavedra and M. Pérez-Victoria, No like-sign tops at Tevatron: Constraints on extended models and implications for the tz asymmetry, Phys. Lett. B 701 (2011) 93 [arXiv:1104.1385] [INSPIRE].

[34] J. de Blas, Electroweak limits on physics beyond the Standard Model, EPJ Web Conf. 60 (2013) 19008 [arXiv:1307.6173] [INSPIRE].

[35] M. Ciuchini, E. Franco, S. Mishima and L. Silvestrini, Electroweak Precision Observables, New Physics and the Nature of a 126 GeV Higgs Boson, JHEP 08 (2013) 106 [arXiv: 1306.4644] [INSPIRE].

[36] ALEPH, DELPHI, L3, OPAL ANd SLD collaborations, LEP Electroweak Working Group, SLD Electroweak Group and SLD Heavy Flavour Group, S. Schael et al., Precision electroweak measurements on the $Z$ resonance, Phys. Rept. 427 (2006) 257 [hep-ex/0509008] [INSPIRE]. 
[37] M. Davier, A. Hoecker, B. Malaescu and Z. Zhang, Reevaluation of the Hadronic Contributions to the Muon $g-2$ and to $\alpha\left(M_{Z}^{2}\right)$, Eur. Phys. J. C 71 (2011) 1515 [Erratum ibid. C 72 (2012) 1874] [arXiv:1010.4180] [INSPIRE].

[38] Particle Data Group collaboration, K.A. Olive et al., Review of Particle Physics, Chin. Phys. C 38 (2014) 090001 [inSPIRE].

[39] ATLAS, CDF, CMS AND D0 collaborations, First combination of Tevatron and LHC measurements of the top-quark mass, arXiv:1403.4427 [INSPIRE].

[40] ATLAS collaboration, Measurement of the Higgs boson mass from the $H \rightarrow \gamma \gamma$ and $H \rightarrow Z Z^{*} \rightarrow 4 \ell$ channels with the ATLAS detector using $25 \mathrm{fb}^{-1}$ of pp collision data, Phys. Rev. D 90 (2014) 052004 [arXiv: 1406.3827] [INSPIRE].

[41] CMS collaboration, Precise determination of the mass of the Higgs boson and tests of compatibility of its couplings with the standard model predictions using proton collisions at 7 and $8 \mathrm{TeV}$, arXiv:1412.8662 [INSPIRE].

[42] CDF AND D0 collaborations, T.E.W. Group, 2012 Update of the Combination of CDF and D0 Results for the Mass of the $W$ Boson, arXiv:1204.0042 [INSPIRE].

[43] ALEPH, CDF, D0, DELPHI, L3, OPAL AND SLD collaborations, LEP and TEvatron Electroweak Working Group, SLD Electroweak and Heavy Flavour Groups, Precision Electroweak Measurements and Constraints on the Standard Model, arXiv: 1012. 2367 [INSPIRE].

[44] ALEPH, DELPHI, L3 AND OPAL collaborations, LEP ELECTROWEAK Working Group, S. Schael et al., Electroweak Measurements in Electron-Positron Collisions at W-Boson-Pair Energies at LEP, Phys. Rept. 532 (2013) 119 [arXiv:1302.3415] [InSPIRE].

[45] J.A. Aguilar-Saavedra, Effective four-fermion operators in top physics: A Roadmap, Nucl. Phys. B 843 (2011) 638 [Erratum ibid. B 851 (2011) 443] [arXiv:1008.3562] [INSPIRE].

[46] I. Dorsner, S. Fajfer and A. Greljo, Cornering Scalar Leptoquarks at LHC, JHEP 10 (2014) 154 [arXiv: 1406.4831] [INSPIRE].

[47] CMS collaboration, Search for quark compositeness in dijet angular distributions from pp collisions at $\sqrt{s}=7 \mathrm{TeV}$, JHEP 05 (2012) 055 [arXiv: 1202.5535] [INSPIRE].

[48] F. del Aguila and J. de Blas, Electroweak constraints on new physics, Fortsch. Phys. 59 (2011) 1036 [arXiv:1105.6103] [INSPIRE].

[49] F. del Aguila, J. de Blas, P. Langacker and M. Pérez-Victoria, Impact of extra particles on indirect $Z^{\prime}$ limits, Phys. Rev. D 84 (2011) 015015 [arXiv:1104.5512] [INSPIRE].

[50] F. del Aguila and J.A. Aguilar-Saavedra, Distinguishing seesaw models at LHC with multi-lepton signals, Nucl. Phys. B $\mathbf{8 1 3}$ (2009) 22 [arXiv:0808.2468] [INSPIRE].

[51] A. Atre, M. Carena, T. Han and J. Santiago, Heavy Quarks Above the Top at the Tevatron, Phys. Rev. D 79 (2009) 054018 [arXiv:0806.3966] [InSPIRE].

[52] A. Atre et al., Model-Independent Searches for New Quarks at the LHC, JHEP 08 (2011) 080 [arXiv: 1102.1987] [INSPIRE].

[53] A. Atre, M. Chala and J. Santiago, Searches for New Vector Like Quarks: Higgs Channels, JHEP 05 (2013) 099 [arXiv: 1302.0270] [INSPIRE].

[54] C. Grojean, E. Salvioni and R. Torre, A weakly constrained $W^{\prime}$ at the early LHC, JHEP 07 (2011) 002 [arXiv:1103.2761] [INSPIRE]. 
[55] J. de Blas, J.M. Lizana and M. Pérez-Victoria, Combining searches of $Z^{\prime}$ and $W^{\prime}$ bosons, JHEP 01 (2013) 166 [arXiv:1211.2229] [INSPIRE].

[56] J.A. Aguilar-Saavedra, R. Benbrik, S. Heinemeyer and M. Pérez-Victoria, Handbook of vectorlike quarks: Mixing and single production, Phys. Rev. D 88 (2013) 094010 [arXiv: 1306.0572] [INSPIRE].

[57] B. Grzadkowski, M. Iskrzynski, M. Misiak and J. Rosiek, Dimension-Six Terms in the Standard Model Lagrangian, JHEP 10 (2010) 085 [arXiv: 1008.4884] [INSPIRE].

[58] W. Buchmüller and D. Wyler, Effective Lagrangian Analysis of New Interactions and Flavor Conservation, Nucl. Phys. B 268 (1986) 621 [InSPIRE].

[59] C. Arzt, M.B. Einhorn and J. Wudka, Patterns of deviation from the standard model, Nucl. Phys. B 433 (1995) 41 [hep-ph/9405214] [INSPIRE].

[60] R. Contino, M. Ghezzi, C. Grojean, M. Muhlleitner and M. Spira, Effective Lagrangian for a light Higgs-like scalar, JHEP 07 (2013) 035 [arXiv: 1303.3876] [INSPIRE].

[61] J. Elias-Miro, J.R. Espinosa, E. Masso and A. Pomarol, Higgs windows to new physics through $D=6$ operators: constraints and one-loop anomalous dimensions, JHEP 11 (2013) 066 [arXiv: 1308.1879] [INSPIRE].

[62] R.S. Gupta, A. Pomarol and F. Riva, BSM Primary Effects, Phys. Rev. D 91 (2015) 035001 [arXiv: 1405.0181] [INSPIRE]. 